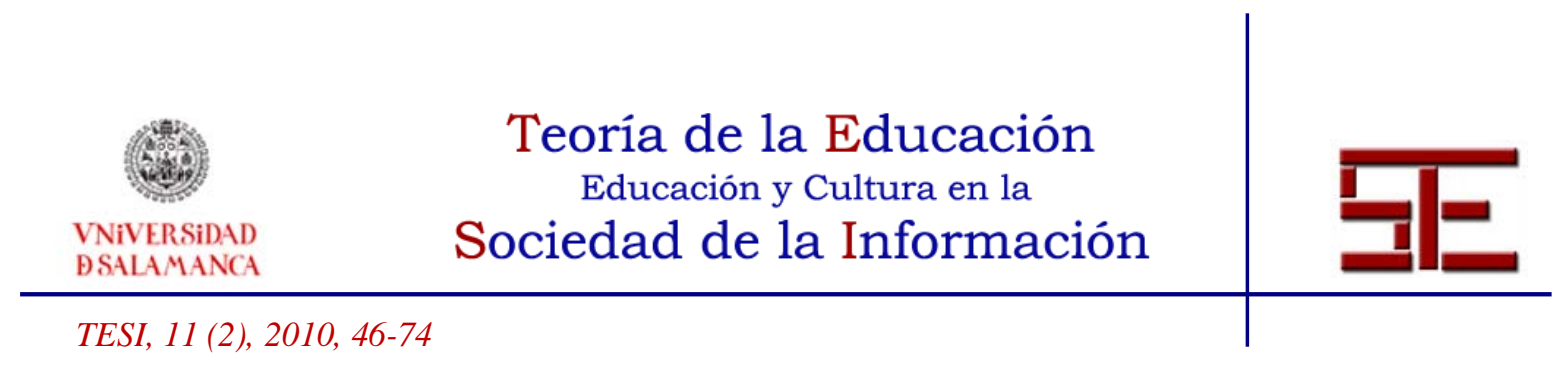

\title{
SIMULACIONES COMPUTACIONALES EN LA ENSEÑANZA DE LA FÍSICA MÉDICA
}

Resumen:

Las universidades europeas están inmersas en un proceso de cambio estructural que tiene como objetivo conseguir mejoras en la calidad de la enseñanza superior. El desarrollo del EEES y la incorporación del uso de las TIC facilitarán la consecución de dicho objetivo. En este marco de referencia, hemos realizado una herramienta multicanal (FISIMED) que permite al estudiante adquirir conocimientos sobre la disciplina "Física Médica" que se imparte en nuestra Facultad. En la actualidad, aunque la plataforma incluye distintos tipos de contenidos, nuestro esfuerzo se dirige especialmente al desarrollo de simulaciones computacionales.

El objetivo fundamental de este trabajo es mostrar las posibilidades que ofrece la tecnología de animación y/o simulación aplicada a los procesos de enseñanzaaprendizaje en la Universidad y, fundamentalmente, al campo de la Física Médica. Se exponen conceptos básicos de su uso, se citan diversas experiencias en el contexto universitario y se analizan las posibilidades que puede tener en la enseñanza de nuestra disciplina.

Palabras clave: Educación Médica, Física Médica, Aprendizaje electrónico, Simulación Computacional, Simulaciones Computacionales en Física.

Francisco Javier Cabrero Fraile, José Miguel Sánchez Llorente, Ana B. Sánchez García, Javier Borrajo Sánchez, $M^{a}$ José Rodríguez Conde, Marta Cabrero Hernández y Juan Antonio Juanes Méndez 


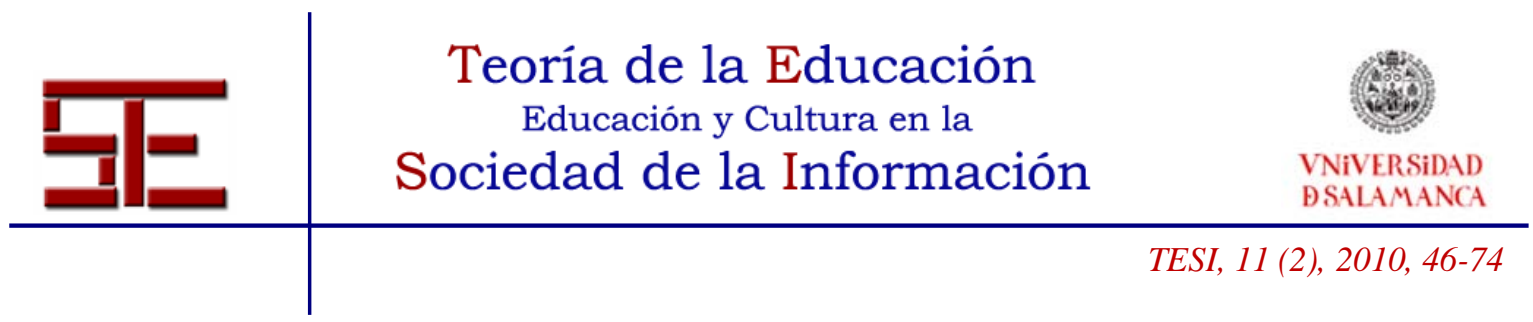

\title{
COMPUTATIONAL SIMULATIONS IN TEACHING MEDICAL PHYSICS
}

\begin{abstract}
:
European Universities are immersed in a process of change that will entail structural changes and which has as an objective the improvement of teaching quality in higher education. The development of the European Space for Higher Education, together with the inclusion of ICTs in the classroom will make it easier to attain the aforementioned objective. In this referential framework, we have developed a multichannel tool (FISIMED) which allows the student to acquire knowledge about the subject 'Medical Physics', taught in the faulty of Medicine at the University of Salamanca. Nowadays, besides the network includes different types of contents, our main effort is focused on the development of computer-based simulations.

The main objective in this research is to show the different possibilities that animation and simulation technologies offer to teaching and learning processes at university, and, more specifically, in the field of Medical Physics. We present basic concepts for its use, quote several experiences carried out at the university context and analyse the different possibilities that the teaching of the aforementioned discipline can have.
\end{abstract}

Key words: Medical Education, Medical Physics, e-Learning, Computer Simulation, Computational Physics.

Francisco Javier Cabrero Fraile, José Miguel Sánchez Llorente, 


\section{SIMULACIONES COMPUTACIONALES EN LA ENSEÑANAZA DE LA FÍSICA MÉDICA}

Francisco Javier Cabrero Fraile

cabrero@usal.es

José Miguel Sánchez Llorente

jmsll@usal.es

Ana B. Sánchez García

asg@usal.es

Javier Borrajo Sánchez

borrajo@usal.es

María José Rodríguez Conde

mjrconde@usal.es

Marta Cabrero Hernández

marta.ch@usal.es

Juan Antonio Juanes Méndez

jajm@usal.es

Universidad de Salamanca

\section{1.- INTRODUCCIÓN}

La Universidad Española está sumida en un proceso de cambio estructural que tiene como objetivo conseguir mejoras en la calidad de la enseñanza superior. La exigencia de una profunda transformación del modelo educativo, centrado en el proceso de enseñanza-aprendizaje, pasa por una atención individualizada que permita cuantificar el grado de esfuerzo del alumno.

El desarrollo del Espacio Europeo de Educación Superior (EEES) y la incorporación y extensión del uso de las Tecnologías de la Información y la Comunicación (TIC) debe favorecer un cambio en el papel y práctica pedagógica del docente. En este sentido, las TIC facilitarán el desarrollo de una acción formativa flexible, centrada en el estudiante y adaptada a sus características y necesidades.

Francisco Javier Cabrero Fraile, José Miguel Sánchez Llorente, Ana B. Sánchez García, Javier Borrajo Sánchez, $M^{a}$ José Rodríguez Conde, Marta Cabrero Hernández y Juan Antonio Juanes Méndez 


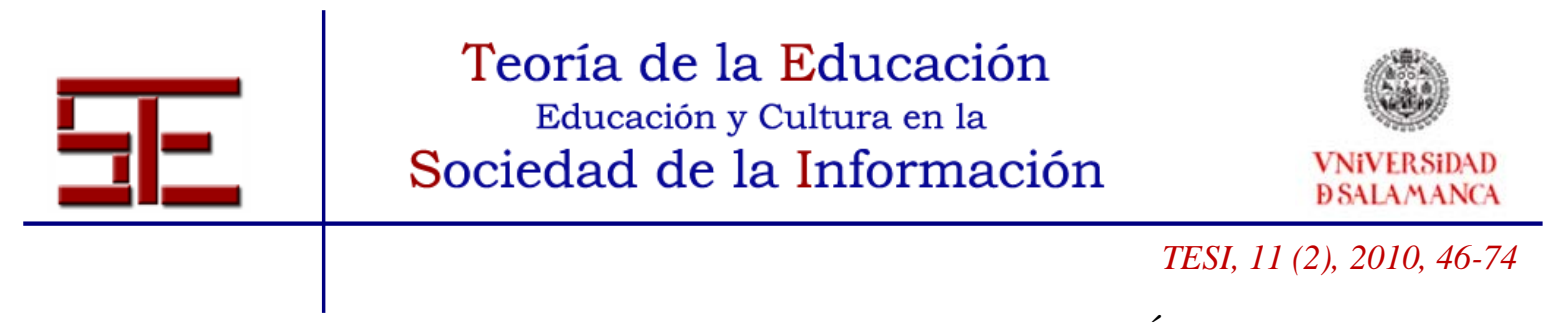

En este marco de referencia, la Unidad de Física Médica del Área de Radiología y Medicina Física del Departamento de Física, Ingeniería y Radiología Médica de la Universidad de Salamanca ha realizado una herramienta multicanal (FISIMED) [LMS; Learning Management System], desarrollada en distinta extensión para tres plataformas (PC, Internet y Móvil), que permite al estudiante adquirir conocimientos personalizados referentes a los contenidos de la disciplina "Física Médica”.

Un LMS es un Sistema de Gestión de Aprendizaje que se emplea para administrar, distribuir y controlar las actividades de formación presencial o e-Learning / Aprendizaje-Electrónico de una institución u organización. Las principales funciones del LMS son gestionar usuarios y recursos, así como materiales y actividades de formación, administrar el acceso, controlar y hacer seguimiento del proceso de aprendizaje, realizar evaluaciones, generar informes y gestionar servicios de comunicación (por ejemplo, foros de discusión). La mayoría de los LMS funcionan con tecnología Internet (páginas web) y, generalmente, se focalizan en gestionar contenidos creados por fuentes diferentes, ya que la labor de desarrollar los contenidos para los cursos se lleva a cabo mediante un LCMS (Learning Content Management Systems).

La Física Médica es la rama de la Física que comprende la aplicación de los conceptos, leyes, modelos, agentes y métodos propios de la Física a la prevención, diagnóstico y tratamiento de las enfermedades, desempeñando una importante función en la asistencia médica, en la investigación biomédica y en la optimización de algunas actividades sanitarias. En la actualidad, de acuerdo con la Sociedad Española de Física Médica (SEFM, 2004), la disciplina:

- Aporta los fundamentos físicos de múltiples técnicas diagnósticas y terapéuticas.

- Proporciona la base científica para la comprensión y desarrollo de las modernas tecnologías que han revolucionado el diagnóstico médico.

- Establece los criterios para la correcta utilización de los agentes físicos empleados en Medicina.

- Sienta las bases necesarias para la medida de las variables biomédicas.

- Aporta los fundamentos necesarios para el desarrollo de modelos que explican el funcionamiento del cuerpo humano.

En la Facultad de Medicina de la Universidad de Salamanca, los contenidos de la disciplina se imparten en las asignaturas "Física Médica" de la Licenciatura de Medicina y "Radiología General, Medicina Física y Física Aplicada” de la Licenciatura de Odontología.

Francisco Javier Cabrero Fraile, José Miguel Sánchez Llorente,

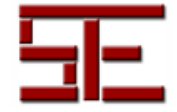
Ana B. Sánchez García, Javier Borrajo Sánchez, $M^{a}$ José Rodríguez Conde, Marta Cabrero Hernández y Juan Antonio Juanes Méndez 


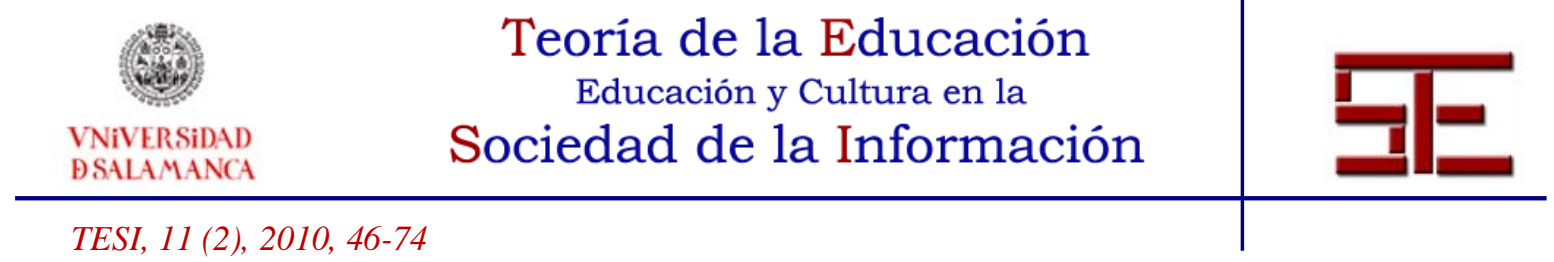

En los últimos decenios se han producido grandes novedades en el campo de la Física Médica, tanto en su desarrollo como en su docencia. El contexto del alumnado está integrado por estudiantes que acceden a la Facultad una vez superado el examen de Selectividad y que, al inicio del curso, no comprenden la necesidad del estudio de la Física. Por tanto, la manera de tratar los temas debe intentar potenciar el interés de los alumnos, para que éstos sepan reconocer la necesidad de aprender lo que se les pretende enseñar.

En este sentido, la utilización de simulaciones informáticas que crean situaciones artificiales que se acercan en alguna medida a la situación real (material de simulación), permite aumentar la motivación de los estudiantes y les proporciona una mejor comprensión de conceptos que, por su complejidad, constituyen una tarea difícil para ellos (Cabrero et al., 2009).

\section{1.- EI Espacio Europeo de Educación Superior.}

La Declaración de Bolonia de 1999 proponía la creación del denominado Espacio Europeo de Educación Superior (EEES) con la finalidad de establecer una armonización de la Educación Superior en Europa. Posteriormente fueron definiéndose objetivos concretos, siendo uno de los principales a alcanzar el que todos los países adopten un sistema flexible, comparable y compatible de titulaciones que facilite la movilidad de estudiantes y titulados. El plazo temporal fijado para su realización finaliza en el año 2010 (Grupo de Promotores de Bolonia en España (Programa Sócrates 2005 - 1678/001 SO2-21BOPR)).

EL EEES intenta aumentar el número de titulados superiores en Europa, eliminar las barreras a la libre circulación de estudiantes y titulados en la UE y en el resto de Europa, hacer de Europa un lugar más atractivo para estudiar o investigar o facilitar el aprendizaje continuado, entre otros. Por otra parte, proyecta impulsar un cambio educativo, facilitando el paso de la enseñanza al aprendizaje autónomo. Por tanto, pretende situar al estudiante en el centro del proceso de aprendizaje para que sea autónomo y capaz de "aprender a aprender". Este cambio debe ser facilitado mediante la utilización en la enseñanza de las tecnologías de la información y comunicación (Bonete, 2006).

En el mismo sentido se manifiesta Cabero (2005), al afirmar que el cambio en las actividades que implica el EEES llevará a una transformación radical del papel del profesor y del alumno pasando, el primero, de actor a diseñador de situaciones y escenarios de aprendizaje, y el segundo, de espectador a actor de su aprendizaje. En esta nueva situación las TIC jugarán un papel muy significativo.

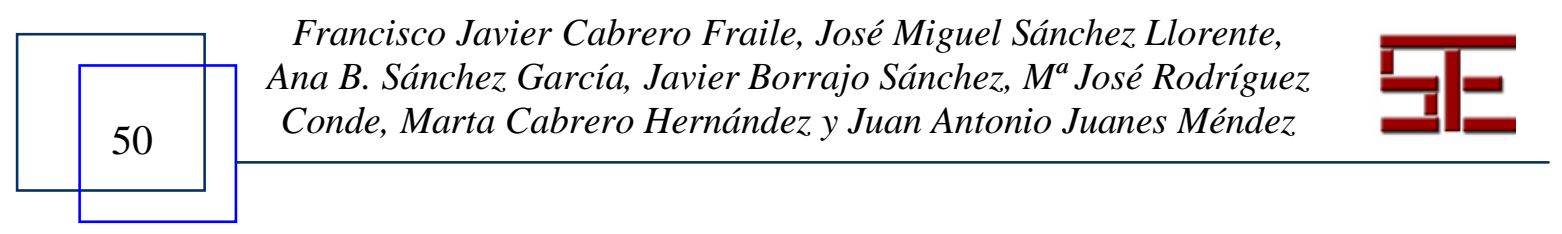




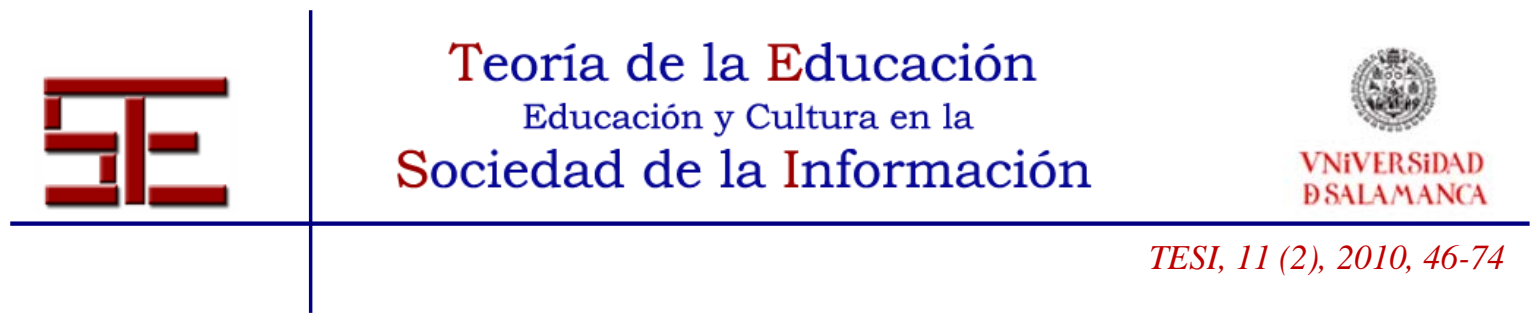

En la actualidad, el EEES constituye una realidad en la universidad española, ya que se encuentra en pleno proceso de implantación. La norma fundamental es el Real Decreto 1393/2007, por el que se establece la ordenación de las enseñanzas universitarias oficiales. Antes de su publicación, las universidades españolas distribuyeron información y organizaron programas de formación sobre el EEES, participaron en programas de innovación docente y asistieron a las reuniones nacionales e internacionales que se convocaron sobre dichos temas. Asimismo, dada la importancia de las tecnologías de la información y la comunicación, diseñaron planes estratégicos en relación con la utilización de la tecnología.

La Comisión para la Renovación de las Metodologías Educativas en la Universidad, constituida en el año 2005 por el Ministerio de Educación y Ciencia, publicó en 2006 un informe sobre propuestas para la renovación de dichas metodologías en el que percibe el proceso de construcción del EEES como "la oportunidad perfecta para impulsar una reforma que no debe quedarse en una mera reconversión de la estructura y contenido de los estudios, sino que debe alcanzar al meollo de la actividad universitaria, que radica en la interacción profesores-estudiantes para la generación de aprendizaje”.

La Universidad de Salamanca, consciente del cambio desde el actual modelo de enseñanza a otro más centrado en el papel activo del estudiante en el proceso de aprendizaje, que implica el desarrollo de nuevas metodologías docentes, y buscando impulsar una docencia de calidad, ha convocado en los dos últimos cursos académicos programas de ayudas para la innovación docente. Entre los ámbitos de actuación de dichas ayudas, se encuentra el diseño y desarrollo de proyectos basados en las TIC.

\section{2.- Las TIC en el marco del EEES.}

La Conferencia de Rectores de las Universidades Españolas publicó en el año 2006 un análisis estratégico sobre las TIC en el Sistema Universitario Español. En dicho estudio, Fernández, Rodeiro y Ruzo (2006) resumen los resultados del informe que la Comisión Europea solicitó a la consultora danesa PLS RAMBOL Management sobre la utilización (2002- 2003) y potencial de las TIC en las universidades europeas (PLS RAMBOL Management, 2004). El informe da una serie de recomendaciones a cuatro niveles, como conclusión a los resultados obtenidos:

- A nivel UE: "las recomendaciones se orientan a la diseminación de las experiencias y buenas prácticas que puedan ayudar a unas universidades a aprovechar la experiencia ya desarrollada por otras".

- A nivel nacional: introducción de las TIC dentro de los planes estratégicos de las universidades (entre otras).

Francisco Javier Cabrero Fraile, José Miguel Sánchez Llorente,

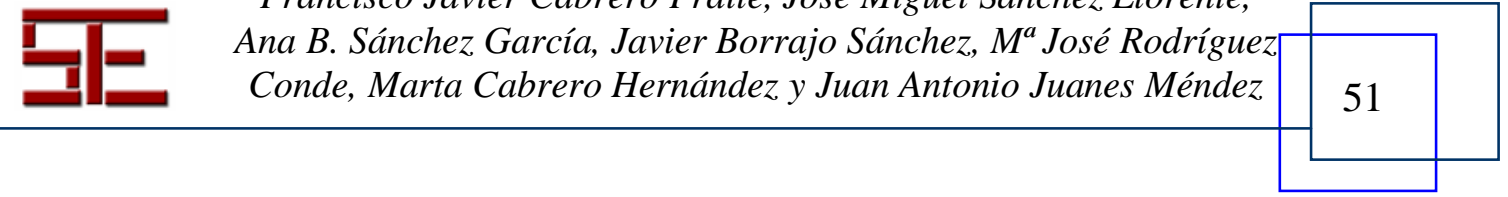




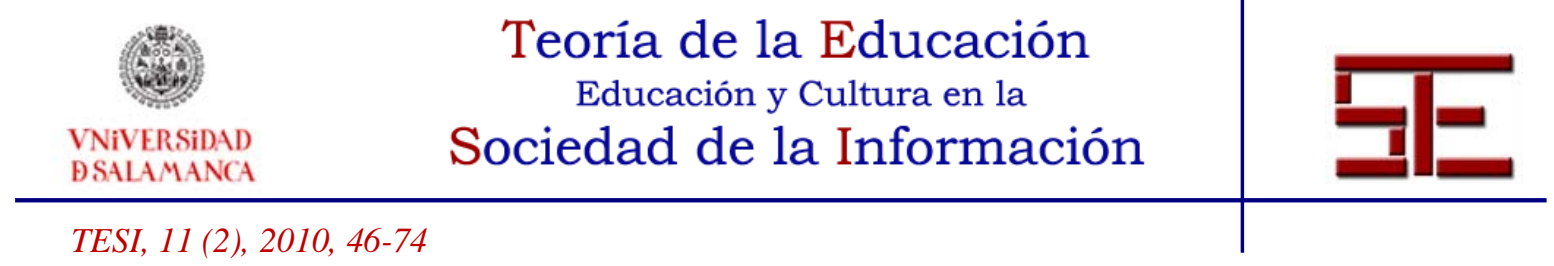

- A nivel universidad, "las recomendaciones se orientan a completar el proceso".

- A nivel de líneas de investigación futuras, se indica la importancia de profundizar en la investigación tanto en el ámbito pedagógico (impacto de las TIC en la interacción profesor-alumno, desarrollo de herramientas TIC, etc.) como organizativo (incremento de la disposición de las universidades hacia la innovación).

El documento de la Conferencia de Rectores de las Universidades Españolas de 2006 ("Las TIC en el Sistema Universitario Español”) señala en su capítulo 5, como primer objetivo del eje "enseñanza-aprendizaje", la incorporación de las TIC a la docencia en las aulas no sólo para formar a profesores y alumnos en el manejo de estas tecnologías, sino para generar una actitud activa y un interés hacia su utilización en las actividades asociadas al proceso de aprendizaje de los alumnos.

Asimismo, marca como segundo y tercer objetivos la necesidad de proporcionar la infraestructura tecnológica necesaria y la de facilitar la docencia virtual mediante iniciativas en formación e implantación de plataformas informáticas (Ruzo y Rodeiro, 2006).

El recorrido de las TIC desde su aparición permite asistir a una panorámica en el mundo de la formación y de la enseñanza que, según Cabero (2005), podríamos describir de acuerdo a los siguientes parámetros:

- Interés de los centros por las nuevas tecnologías.

- Presencia de los medios informáticos en los centros.

- Uso curricular: admisión, en general, por parte de los profesores de las ventajas de la utilización de las TIC y de la necesidad de la propia formación en las nuevas tecnologías.

- Adecuación de los medios a las características del centro.

- Formación del profesorado del centro respecto al uso de medios basados en las TIC.

- Participación en proyectos de incorporación de las TIC a los centros (innovación e investigación).

En el marco del EEES, las TIC proporcionarán recursos que van a permitir a las universidades españolas mejorar la calidad de la enseñanza, ya que los docentes podrán ofrecer una formación que otorgue al estudiante un mayor protagonismo en su proceso de aprendizaje. Según Marquès (2006), las tres claves principales que permitirían asegurar el éxito en el cambio hacia nuevas metodologías docentes con apoyo en las TIC son: disponer de los recursos necesarios que permitan implicar al conjunto del profesorado en los cambios, proporcionar a los docentes la formación adecuada en el

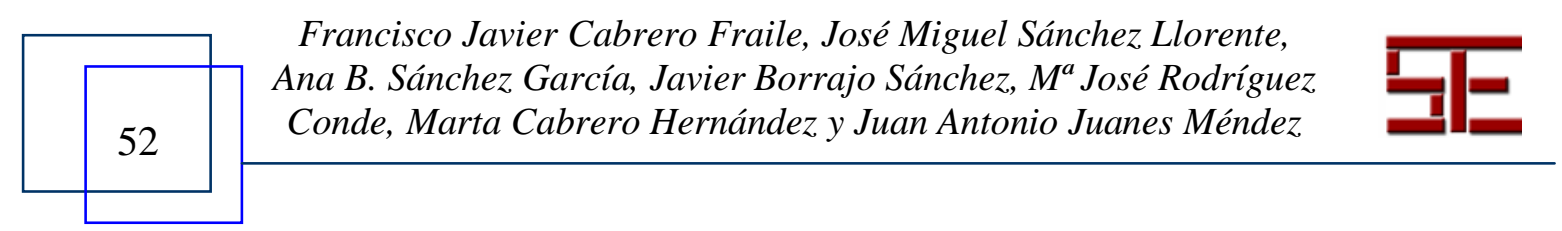




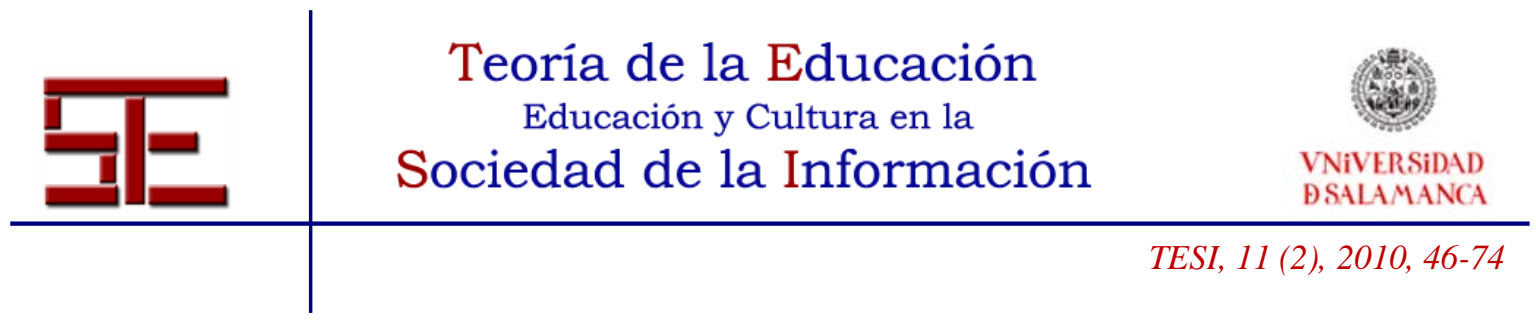

uso de las nuevas tecnologías y generar en el profesorado la motivación necesaria para percibir el cambio como conveniente.

Por otra parte, la implantación de los nuevos planes de estudio obligará a los alumnos a asumir una nueva concepción del aprendizaje en el que deberán trabajar con menos dependencia del profesor. Por tanto, el conocimiento de las herramientas TIC básicas se hará absolutamente necesario (procesador de textos, tratamiento de imágenes, editor de presentaciones, uso de navegadores y buscadores de Internet, correo electrónico, plataformas de campus virtual, etc.). Los distintos órganos de gobierno deberán realizar las actuaciones necesarias para que estas condiciones puedan cumplirse (Marquès, 2006).

Una de sus repercusiones más significativas de las tecnologías de la información y comunicación para las universidades va a ser la implantación y extensión de la formación a distancia (Cabero, 2005). Ortega et al. (2002) presentaron una comunicación en el II Congreso Internacional “Docencia Universitaria e Innovación” en la que hacían referencia al nuevo modelo de formación del profesorado universitario basado en la progresiva implantación de las TIC y especialmente de plataformas virtuales para la docencia/aprendizaje. En concreto, se referían al desarrollo de EUDORED (Entorno de la Universidad de Salamanca para la docencia en red), germen de la actual plataforma virtual de aprendizaje de nuestra Universidad.

En la actualidad, la formación telemática es una realidad en todas las instituciones universitarias, hasta el punto en el que las nuevas tecnologías de la información, y sobre todo las telemáticas, han transformado la Universidad.

\section{2.- OBJETIVOS}

La plataforma informática FISIMED está diseñada para un crecimiento progresivo, aunque los contenidos implementados en la misma incluyen ya información sobre la materia, presentaciones PowerPoint, software multimedia, galerías de imágenes y vídeos, test de autoevaluación y animaciones o simulaciones, entre otros. En la actualidad, nuestro esfuerzo se dirige especialmente al diseño, desarrollo e implementación de animaciones y simulaciones computacionales en el Sistema de Gestión de Contenidos (CMS) de apoyo al proceso de enseñanza-aprendizaje online de la Física Médica.

El objetivo fundamental de este trabajo es mostrar las posibilidades que ofrece la tecnología de animación y/o simulación aplicada a los procesos de enseñanzaaprendizaje en el campo de las Ciencias de la Salud y, en especial, en el campo de la

Francisco Javier Cabrero Fraile, José Miguel Sánchez Llorente, Ana B. Sánchez García, Javier Borrajo Sánchez, Ma José Rodríguez
Conde, Marta Cabrero Hernández y Juan Antonio Juanes Méndez 


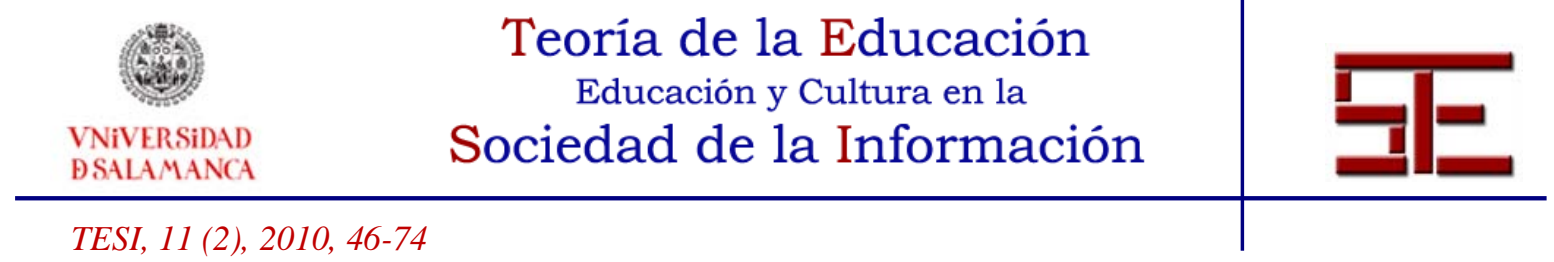

Física Médica. En el artículo se exponen los conceptos básicos del uso de esta tecnología, se citan diversas experiencias en el contexto universitario y se analizan las posibilidades que puede tener en la enseñanza de nuestra disciplina.

No obstante, antes de abordar el estudio del tema enfocando el mismo hacia el desarrollo de simulaciones computacionales en la enseñanza de una materia de pregrado como es la Física Médica, haremos referencia a uno de los objetivos marcados por la Unión Europea, la potenciación de las TIC y su aplicación en el campo de la Sanidad en general.

\section{3.- INNOVACIÓN DOCENTE A TRAVÉS DE LAS TIC EN EL CAMPO DE LA EDUCACIÓN MÉDICA}

La revista Educación Médica publicó, en diciembre de 2006, un número extraordinario [Innovación docente vía TIC. Fundación IAVANTE. Educación Médica, 9 (supl. 2)] cuyos contenidos corresponden a los temas expuestos en el II Symposium Internacional IAVANTE 2005. Este Symposium examinó en profundidad el e-Learning y secundariamente la telemedicina, dada la íntima conexión entre ambas (Blanco y Vázquez, 2006; Vázquez y Blanco, 2006).

Joseph M. Duart, Director de la Cátedra UNESCO de e-Learning de la Universitat Oberta de Catalunya, define el término e-Learning como "una metodología de enseñanza aprendizaje basada en el uso de contenidos educativos distribuidos a través de un soporte electrónico, fundamentalmente Internet” (Duart, 2006). Dicho de otra forma, "acceso online a recursos de aprendizaje, desde cualquier sitio y a cualquier hora” (Holmes y Gardner, 2006). En definitiva, se trata de relacionar tres ideas clave: metodología de enseñanza y de aprendizaje, contenidos educativos y potencial de acceso a la información vía Internet (Duart, 2006).

En el caso de la educación médica, e-Learning representa una modalidad en plena expansión de enseñanza y aprendizaje a distancia basada en las TIC, que sustituirá a la formación presencial en muchos campos relacionados con las Ciencias de la Salud. No obstante, la necesidad de estudios de investigación que permitan identificar el proceso óptimo de desarrollo e implementación de las plataformas constituye para Vázquez et al. (2006) la base de su aceptación en el campo de la educación médica.

En este sentido, según Riley et al. (2006), el e-Learning puro estaría menos indicado en un ámbito como el de la educación médica que su uso en combinación con otras estrategias docentes ("blended learning”). El B-Learning entendido como la suma de autoestudio, teletutorización y sesiones presenciales tiene como objetivo básico la

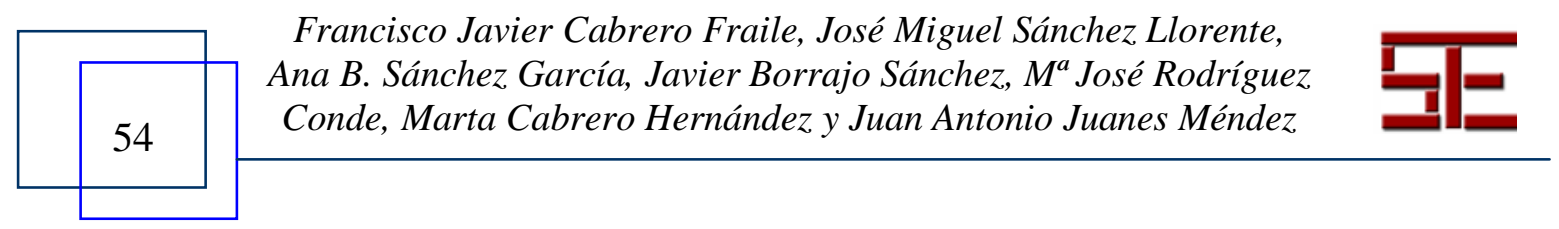




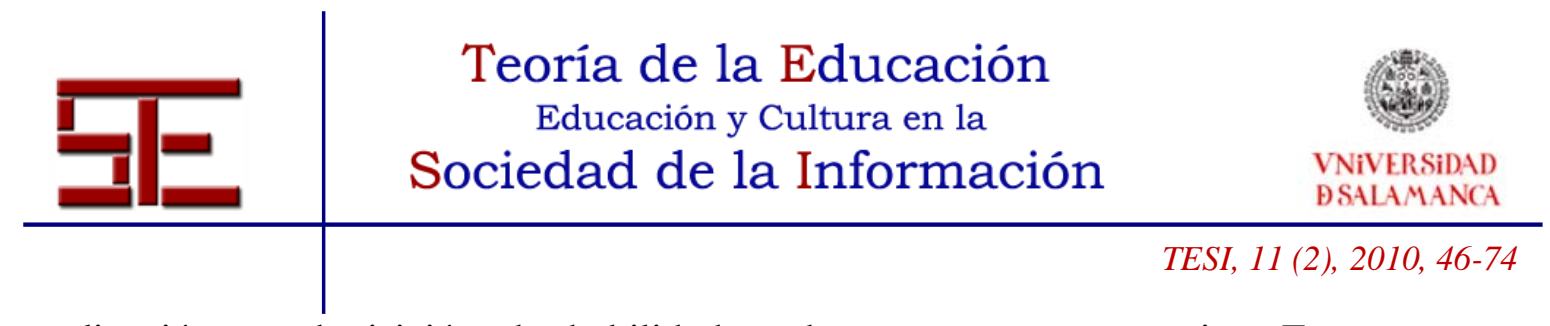

aplicación o adquisición de habilidades, destrezas y competencias. Estos autores muestran algunos ejemplos de su utilización en el campo de la educación médica, combinando una fase no-presencial, donde se tratan conceptos teóricos, y una fase presencial:

- Combinación de e-Learning (fase no presencial mediante material didáctico en formato CD interactivo) y metodología presencial.

- Combinación de e.Learning con nuevas metodologías de simulación que se utilizan fundamentalmente para intentar reproducir un ambiente similar a la realidad que tendrá que enfrentar el alumno o el profesional sanitario (situaciones complejas, análogas a las que tendrán que resolver en su actividad asistencial):

- Simulación de realidad virtual: mediante la utilización de simuladores donde se pueden entrenar habilidades manuales, adquirir conocimientos teóricos y entrenar la competencia en toma de decisiones. Por ejemplo, simuladores de realidad virtual en técnicas endoscópicas o laparoscópicas, entre otros.

- Simulación robótica: mediante maniquíes completos que simulan pacientes. La importancia de la simulación robótica en el entrenamiento clínico se basa en la posibilidad de trabajar en procesos asistenciales (patología respiratoria, cardíaca, etc.), en situaciones muy próximas a la realidad.

- Simulación escénica (con actores entrenados): con la metodología de simulación escénica se pretende, en las fases no presencial y presencial, que "el profesional/alumno consiga, principalmente, conocimientos teóricos y competencias de habilidades relacionales y el manejo de situaciones difíciles sobre todo en el manejo de la anamnesis, la exploración física, la información al paciente y familiares y la resolución de conflictos" (Riley et al., 2006).

En resumen, en relación con el entrenamiento de profesionales sanitarios, opinan que el futuro está en el "blended learning”, mediante una combinación efectiva e-Learning y metodologías de simulación innovadoras.

Actualmente existen tres grupos de opciones para la selección de una plataforma tecnológica de aprendizaje a través de las TIC: elaborar una plataforma propia, contratar un producto de mercado o utilizar una plataforma elaborada bajo los criterios de "software libre". En general, las instituciones se inclinan por el uso de plataformas de “software libre” (Lago, 2006). En cualquier caso, la plataforma tecnológica no es lo más importante, ya que los contenidos o materiales y el papel a jugar por el profesor o el

Francisco Javier Cabrero Fraile, José Miguel Sánchez Llorente,

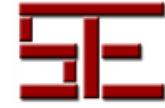

Ana B. Sánchez García, Javier Borrajo Sánchez, Ma José Rodríguez Conde, Marta Cabrero Hernández y Juan Antonio Juanes Méndez 


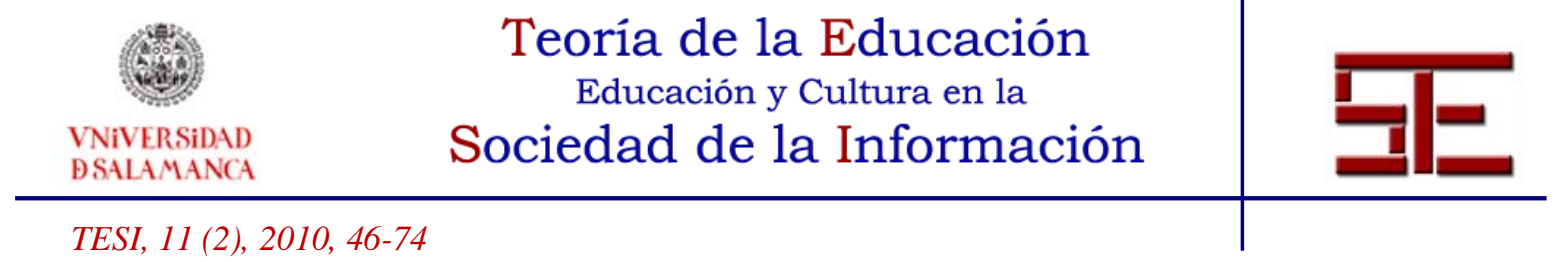

tutor tienen una mayor relevancia. En este sentido, con respecto a los contenidos, la tendencia es a elaborar materiales basados en animaciones y a introducir simulaciones de determinadas operaciones (Fundación IAVANTE, 2006).

En la Universidad de Salamanca, en el año 2009, se procedió a la lectura y defensa pública de la Tesis Doctoral "Diseño, codificación y puesta en valor de una plataforma multicanal: Sistema de Gestión de Aprendizaje (L.M.S.), para la ayuda en la enseñanza individualizada de fundamentos físicos aplicados a la Medicina”. El objetivo fundamental de este trabajo de investigación fue diseñar, desarrollar, implementar y evaluar una herramienta informática de apoyo al proceso de enseñanza-aprendizaje de "Física Médica" que se imparte en la Facultad de Medicina de nuestra Universidad (FISIMED). En definitiva, se pretendió realizar una plataforma multicanal que permitiera al estudiante adquirir conocimientos personalizados de forma interactiva, referentes a los contenidos de nuestra disciplina.

Algunos resultados de la investigación educativa llevada a cabo se muestran en otro de los artículos que componen esta monografía [Sánchez-Llorente et al.]. Asimismo, los contenidos implementados hasta el curso académico 2008-2009 fueron resumidos en un artículo presentado en el XVII Congreso Nacional de la Sociedad Española de Física Médica (SEFM) y XII Congreso Nacional de la Sociedad Española de Protección Radiológica (SEPR) (Cabrero et al., 2009).

\section{4.- LAS SIMULACIONES EN EDUCACIÓN MÉDICA}

En el apartado anterior, hemos hecho referencia a la educación médica basada en simulaciones (EMBS) citando algunas de las modalidades utilizadas. La EMBS se presenta como una herramienta de extraordinario valor para mejorar la calidad del aprendizaje clínico y, por consiguiente, la seguridad del paciente dado el preocupante panorama relacionado con la magnitud de los errores médicos en los sistemas de atención sanitaria. En la actualidad, los avances en los campos de la simulación médica y de las tecnologías virtuales han permitido disponer de una serie de productos que Ziv y Berkenstadt (2008) resumen en los siguientes puntos:

- Estimuladores de baja tecnología. Modelos o maniquíes plásticos utilizados para practicar habilidades físicas.

- Pacientes simulados. Actores entrenados para desempeñar el papel de pacientes.

- Animales vivos y cadáveres. Utilizados en el entrenamiento de procedimientos quirúrgicos.

- Simuladores informáticos. Programas informáticos para evaluar el conocimiento clínico y la toma de decisiones.

Francisco Javier Cabrero Fraile, José Miguel Sánchez Llorente,

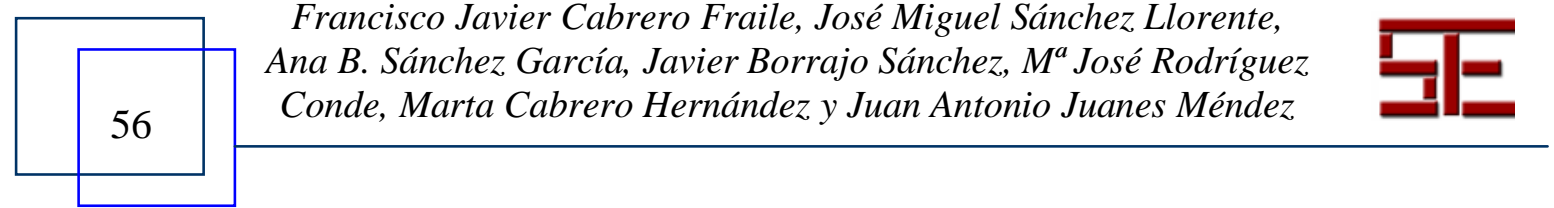




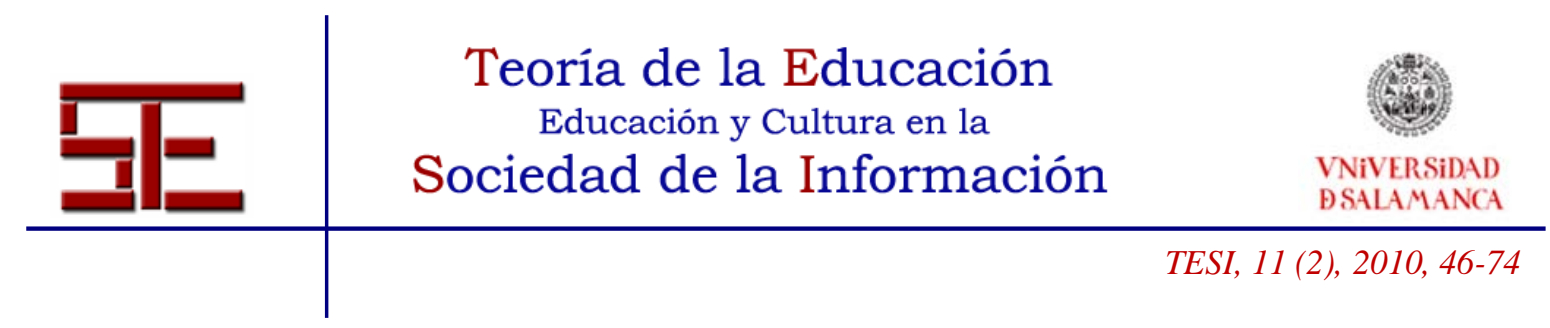

- Entrenadores en tareas complejas. Simuladores informáticos sofisticados que reproducen tareas clínicas como la ecografía, la colonoscopia, la broncoscopia o la cirugía laparoscópica.

- Simuladores de pacientes informatizados realistas. Maniquíes de cuerpo entero regidos por ordenador que permiten el entrenamiento en situaciones clínicas complejas en condiciones similares a la vida real.

- Modelos híbridos que utilizan varias de las modalidades comentadas para aumentar la sensación de realidad.

En resumen, la integración de la EMBS en la formación de los estudiantes y de los profesionales sanitarios conducirá a una asistencia sanitaria más segura y ética de los pacientes (Ziv y Berkenstadt, 2008) (Ziv et al., 2003).

En relación con la formación de pregrado, fundamentalmente de los últimos cursos de la licenciatura en medicina, Galindo y Visbal (2007) concluyen que la simulación como herramienta para la educación médica permitirá preparar al estudiante de forma idónea para el contacto directo con el paciente.

Vázquez-Mata (2007) presentó en el Forum Miriam Friedman, dedicado a las simulaciones en educación médica, una ponencia sobre modelos, estrategias y tendencias en España de la simulación en medicina en la que planteaba la necesidad ineludible de una renovación en las metodologías de formación y entrenamiento, así como la utilización de las TIC. En España, destaca cuatro experiencias con distinto enfoque en cuanto a la simulación:

- La Fundación IAVANTE, de la Consejería de Salud de Andalucía, que cubre tanto el entrenamiento del área médico / enfermero como la del área quirúrgica.

- El Centro de Cirugía de Mínima Invasión “Jesús Uson” (Cáceres), dedicado al entrenamiento en nuevas tecnologías quirúrgicas.

- El Centro de Entrenamiento de Situaciones Críticas del Hospital Marqués de Valdecilla de Cantabria.

- El Institut d’Estudis de la Salut, de la Consejería de Salud de Cataluña, que utiliza la simulación con actores entrenados en la evaluación y acreditación de competencias profesionales.

En cualquier caso, comenta las barreras existentes para la incorporación de la simulación en nuestro país: no-existencia de la misma en los curricula de los estudiantes de medicina, ni en la formación de especialistas; coste de los equipos; falta de un rediseño adecuado de hospitales y facultades de medicina en sus estructuras de apoyo a

Francisco Javier Cabrero Fraile, José Miguel Sánchez Llorente,

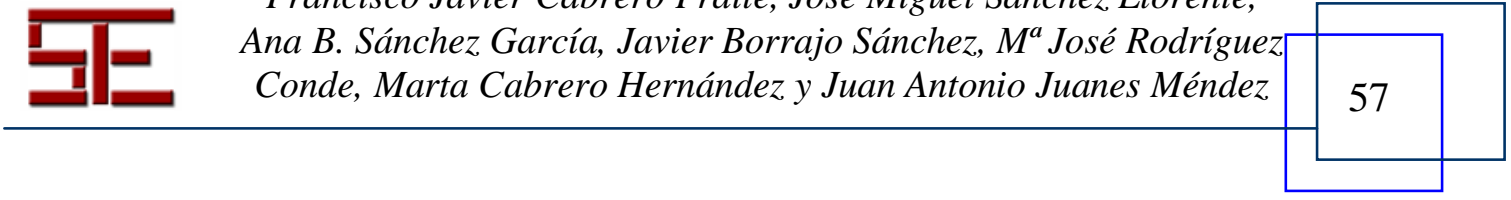




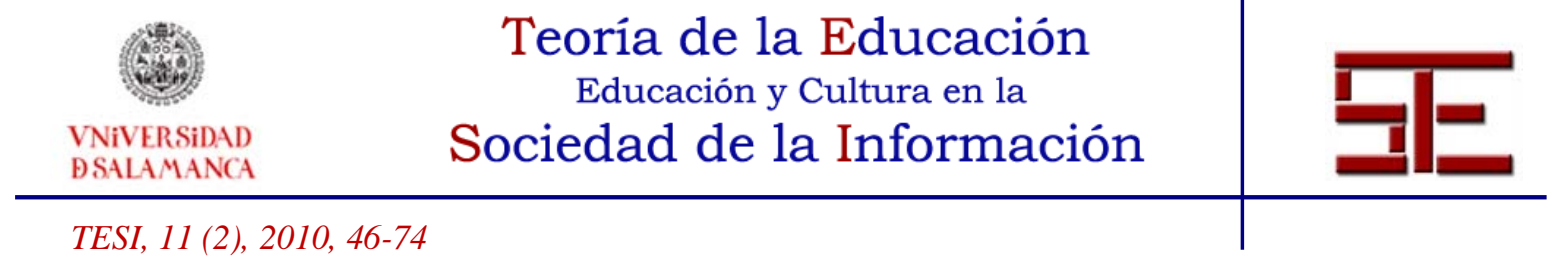

la formación; preparación específica de los profesores; y falta de reconocimiento que tiene el esfuerzo docente de los profesionales sanitarios, entre otros.

En un artículo publicado por Educación Médica en 2008, G. Vázquez-Mata resumía las herramientas de simulación de la realidad como herramientas de cambio que deben implementarse en las facultades de medicina (equipos de robótica, realidad virtual, actores entrenados, etc.), así como el papel que deben jugar las TIC en el entrenamiento básico y avanzado de los alumnos.

En la actualidad, aunque siguen existiendo muchas de las dificultades que hemos comentado, la incorporación de las TIC y la implementación de distintas modalidades de simulación es ya, en mayor o menor grado, una realidad en muchos de nuestros centros. A modo de ejemplo, y refiriéndonos a la enseñanza de pregrado, iCell es un proyecto de investigación en electrofisiología, basado en el desarrollo de una plataforma web interactiva, en el que se han implementado simulaciones computacionales interactivas (applets de Java) para la enseñanza y el aprendizaje de dicha disciplina. iCell es utilizado en la docencia de grado y de postgrado en diferentes países sobre la base de una web interactiva dentro de un entorno colaborativo (Demir y Velipasaoglu, 2003; Demir, 2005).

\section{SIMULACIONES COMPUTACIONALES EN LA ENSEÑANZA DE LA FÍSICA MÉDICA Y DE LA FÍSICA EN GENERAL}

La investigación en educación en la Física, con la puesta en práctica de distintas estrategias de aprendizaje, tiene más de treinta años (McDermott y Redish, 1999). Desde entonces, y sobre todo en la última década, el uso de las TIC ha supuesto un extraordinario impulso en el desarrollo de estas estrategias educativas. El desarrollo de simulaciones computacionales en todas las ramas de las ciencias y, entre ellas la Física, permite al alumno una aproximación mayor a la comprensión del fenómeno que intentamos explicar, ya que existe un gran conjunto de tópicos de esta ciencia que no pueden ser visualizados en el aula por los estudiantes.

\section{1.- Generalidades: Animaciones y/o Simulaciones.}

Las animaciones y simulaciones proporcionan una representación interactiva de la realidad que permite a los estudiantes descubrir cómo funciona o cómo se comporta un fenómeno. El uso de este tipo de herramienta educativa hace posible que el alumno manipule un modelo de la realidad y visualice los efectos producidos mediante un proceso de ensayo-error.

Francisco Javier Cabrero Fraile, José Miguel Sánchez Llorente, Ana B. Sánchez García, Javier Borrajo Sánchez, $M^{a}$ José Rodríguez Conde, Marta Cabrero Hernández y Juan Antonio Juanes Méndez

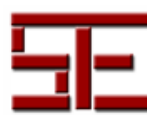




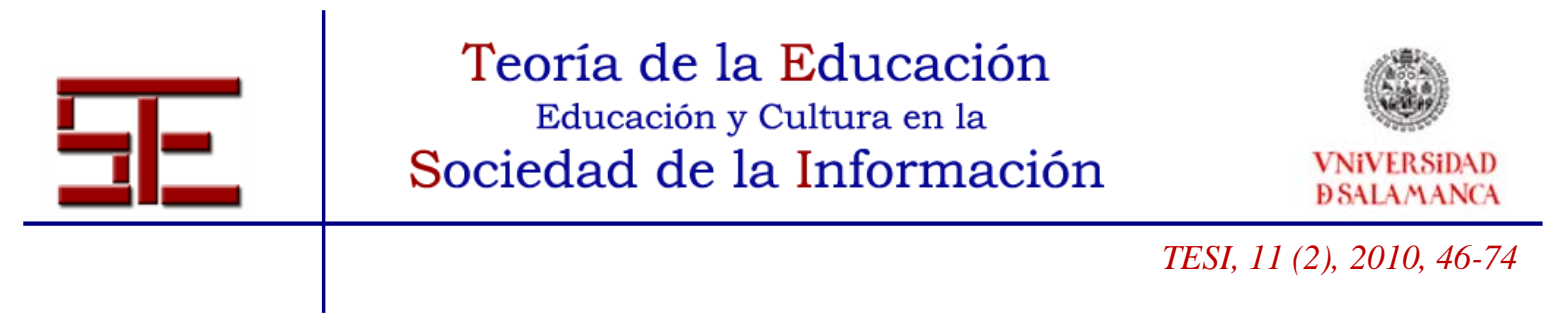

La diferencia básica entre ambos conceptos radica en que los programas de animación pretenden crear efectos artísticos y, por consiguiente, el movimiento que generan es libre. A una animación se asocian, generalmente, contenidos que no precisan de una carga de movimientos basada en trayectorias gobernadas por ecuaciones. Por el contrario, los programas de simulación pretenden crear un efecto real, es decir, traducir al máximo los movimientos reales que ejecuta un objeto. Por tanto, el movimiento será siempre limitado e intentará ajustarse en lo posible a la cinemática real, en detrimento de aspectos visuales o de generalizaciones en el mismo.

El procedimiento habitual para producir un movimiento en animación es realizar escenas en diferentes intervalos de tiempo, de manera que sea el ordenador el que genere la trayectoria a partir de las distintas fases trazadas. Por ejemplo, puede ilustrarse una pelota cayendo, dibujándola a distintas alturas en tiempos distintos. La trayectoria quedará generada por el software de animación, como interpolación de los distintos fotogramas.Sin embargo, en la realización de un movimiento de simulación es preciso definir una estructura (en nuestro ejemplo, las características físicas de la pelota), unas condiciones iniciales (altura del lanzamiento), unas condiciones de contorno (gravedad, ausencia de viento y rozamiento,...) y una ley que gobierne dicho movimiento (ecuaciones de movimiento de un sólido rígido en un campo gravitatorio). De esta forma, se pueden reproducir con exactitud los movimientos del cuerpo en cuestión y estudiar como la variación de los parámetros introducidos afecta a la simulación. Así, por ejemplo, el alumno puede estudiar las variaciones en la caída de un objeto que tenga el doble de peso que la pelota, o el resultado en la caída de ésta cuando se encuentra sujeta a un muelle.

Aunque una de las estrategias educativas seguidas por diversos autores es la construcción de modelos de simulación de fenómenos físicos mediante el uso de distintos lenguajes de programación como, por ejemplo, "pyton” (Rojas y Morales, 2009), el desarrollo de animaciones, o mejor aún, de simulaciones donde el estudiante puede interactuar con el programa, basadas en el lenguaje de programación Java (applets), es desde hace más de una década un estándar en el campo de la Física, hasta el punto de que a aquellas que están diseñadas en el contexto de esta ciencia se las conoce como physlets.

En este sentido, aunque existen en el mercado otras herramientas de software para la codificación, pueden considerarse dos categorías de simulaciones:

- Simulaciones programadas en "Java” (applets - para distinguirlos de los servlets que se ejecutan en servidor) que pueden visualizarse por medio de cualquier navegador, cuando se insertan en una página web, con el único requisito de tener

Francisco Javier Cabrero Fraile, José Miguel Sánchez Llorente,

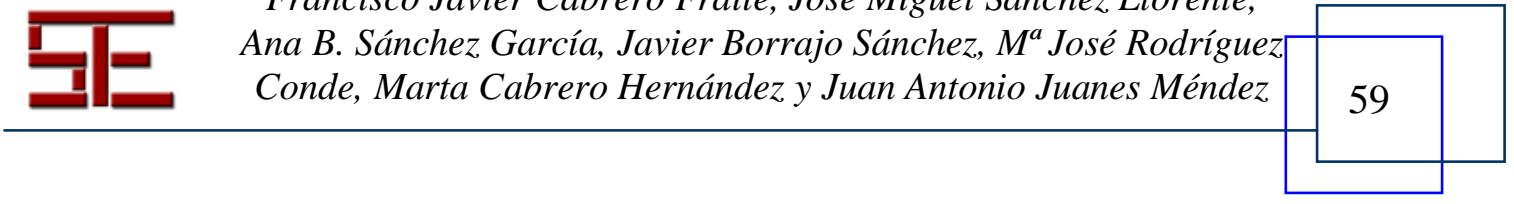




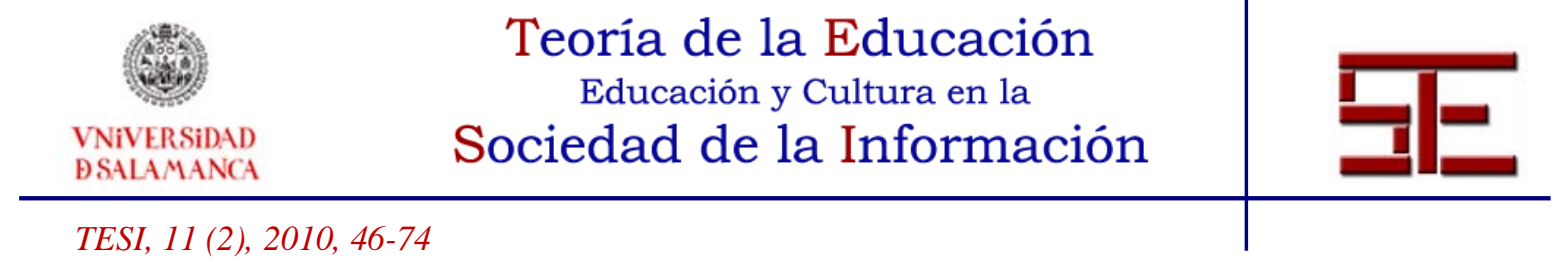

instalada la Máquina Virtual de Java. Según la página oficial de Sun Microsystems (http://www.sun.com/), el software de Java para el equipo o el entorno de ejecución Java se denomina también ejecución Java, entorno de ejecución, JRE, máquina virtual Java, máquina virtual, Java VM, JVM, VM o descarga de Java. La ventaja de Java sobre otros lenguajes de programación es su portabilidad, gracias a que la "Máquina Virtual" se encuentra a un nivel superior al hardware donde funciona la aplicación. Esto quiere decir que cualquier programa Java se ejecutará en la máquina virtual, que será la que convierta el código bytecode al código nativo del dispositivo que se esté utilizando. De esta forma, un programa escrito bajo plataforma Windows puede ser ejecutado en entorno Linux (de ahí el famoso axioma que sigue a Java, "escríbelo una vez, ejecútalo en cualquier parte").

- Simulaciones realizadas en "Adobe Shockwave" que requieren la instalación previa de un "plugin” que permite a los navegadores web la reproducción de los contenidos interactivos. Generalmente estas simulaciones se programan bajo Macromedia Flash, un programa de edición multimedia desarrollado inicialmente por Macromedia y distribuido actualmente por Adobe Systems, que cuenta con una doble faceta (gráfica y de script), de manera que sus ejecutables pueden ser interpretados por un navegador o un sistema operativo sin salida GUI (Interfaz Gráfica de Usuario). Los programas generados suelen ser animaciones, de figuras o texto, e incluso vídeos, pues el formato resultante tiene menor "peso" (menor tamaño) que el original.

En cualquier caso, se dispone de otro tipo de software (“3D Studio Max” y "Maya”, entre otros), más dirigido hacia la simulación, que cuenta con funcionalidades que permiten realizar desarrollos mucho más complejos.

3D Studio Max de Autodesk permite crear modelos en tres dimensiones, animarlos y renderizarlos. Entre sus características dispone de deformadores espaciales (como, por ejemplo, viento, gravedad y vórtice) que se pueden enlazar a los sistemas de partículas para generar condiciones del espacio, o consigue añadir fuerzas personalizadas con una amplia API de dinámica. La herramienta hace posible la realización de simulaciones en el campo de la Matemática, la Física, la Química, las Ciencias de la Salud, etc.

Maya, también de Autodesk, es menos conocido en España. Se utiliza por sus mejoras con respecto al anterior en animaciones relacionadas con el cine. Es superior a 3D Studio Max (más orientado a la infoarquitectura) en el sentido de versatilidad, flexibilidad y adaptabilidad a flujos de trabajo dispares. Además, cuenta con un abanico de herramientas más avanzadas en todas las áreas de trabajo: modelado, animación, dinámicas, etc. En definitiva, es más amplio y adaptable aunque, por otro lado, su curva de aprendizaje es más pronunciada al contar con muchas más funcionalidades.

Francisco Javier Cabrero Fraile, José Miguel Sánchez Llorente,

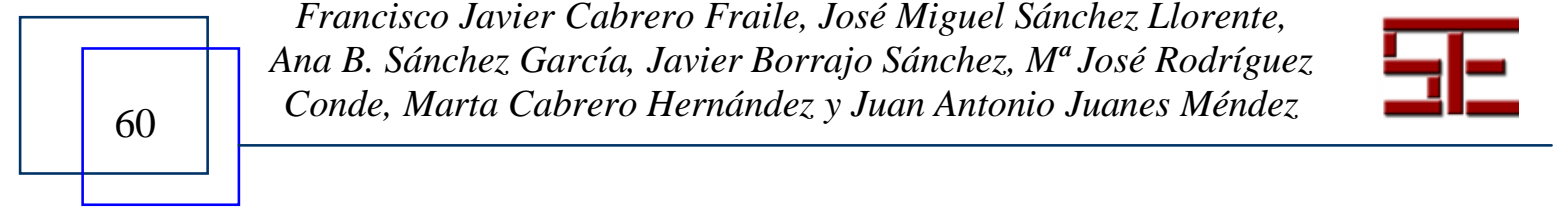




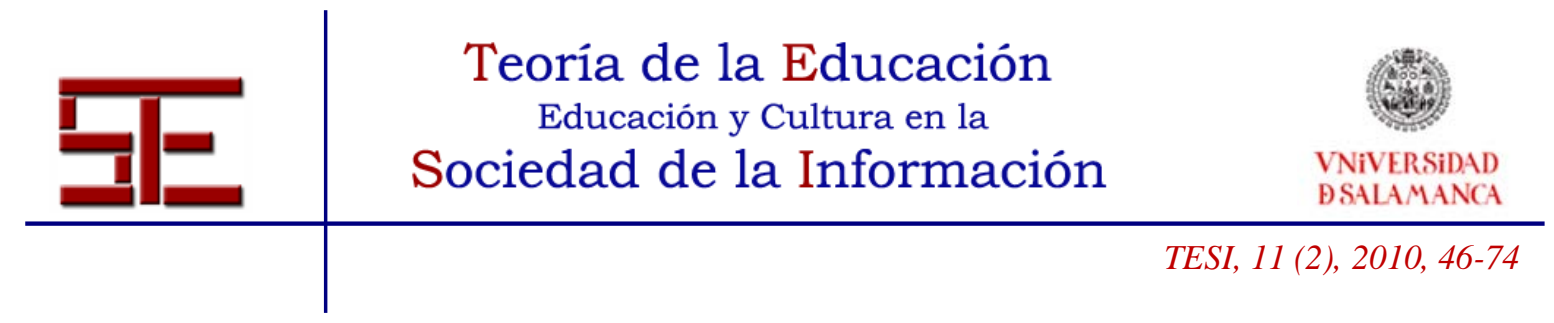

\subsection{La simulación como estrategia educativa en Física Médica.}

En la introducción de este trabajo, en relación con la enseñanza de la Física Médica, hacíamos referencia a la necesidad de tratar los temas intentando potenciar el interés de los alumnos, para que éstos sepan reconocer la necesidad de aprender lo que se les pretende enseñar. En este sentido, señalábamos que la utilización de simulaciones informáticas permite aumentar la motivación de los estudiantes y les proporciona una mejor comprensión de conceptos que constituyen una tarea difícil para ellos.

En la bibliografía encontramos numerosas referencias sobre el uso de animaciones y/o simulaciones en el campo de la Radiología y de la Física Médica. Monsky et al. (2002) presentaron los resultados de un estudio que pretendía evaluar la efectividad de un simulador para ultrasonografía en la formación de residentes de Radiodiagnóstico. Dicho trabajo llevó a la creación de un programa de entrenamiento cuyos resultados pudieron traducirse directamente en una mejor atención al paciente. Por otra parte, el Laboratorio de Física Médica (U 703 Inserm) (Lille - France) viene desarrollando desde hace años una actividad investigadora innovadora en Ingeniería Biomédica, en relación con procedimientos intervencionistas asistidos por imagen médica y técnicas de simulación en Cirugía, Radiología o Radioterapia, entre otras (Vermandel et al., 2007).

La Física Médica y la Ingeniería Biomédica se encuentran entre las primeras profesiones que desarrollaron y aplicaron técnicas de e-Learning en el campo de las Ciencias de la Salud como, por ejemplo, la aplicación de simulaciones (Tabakov, 2008). Un artículo especial sobre el tema fue publicado por el Journal of Medical Engineering and Physics hace unos años (Tabakov, 2005). En este trabajo, el autor especifica una serie de elementos clave para la introducción y uso del e-Learning en "Medical Engineering and Physics” y, entre éstos, los siguientes:

- Ofrece una rápida y fácil actualización de materiales de aprendizaje, algo que es muy importante en una profesión tan dinámica.

- Proporciona una adecuada vía para la resolución de problemas a través de la comprensión de modelos físicos complejos. Por ejemplo, utilizando simulaciones interactivas se consigue aumentar la efectividad en el proceso de aprendizaje.

En relación con la programación de simulaciones específicas, Tavakov (2008) señala que, aunque se trata de herramientas de enseñanza muy eficaces, son muy difíciles de producir, requieren de mucho tiempo para su desarrollo y, a menudo, precisan del conocimiento de varios programas de software, así como de la capacidad de los desarrolladores para presentar la simulación de una manera pedagógica adecuada. Hace referencia al ciclo de vida relativamente corto de las simulaciones, dadas las actualizaciones permanentes de las plataformas de software, como uno de los problemas específicos de las mismas y, finalmente, enumera algunos ejemplos en el campo de la

Francisco Javier Cabrero Fraile, José Miguel Sánchez Llorente, Ana B. Sánchez García, Javier Borrajo Sánchez, Ma José Rodríguez
Conde, Marta Cabrero Hernández y Juan Antonio Juanes Méndez 61 


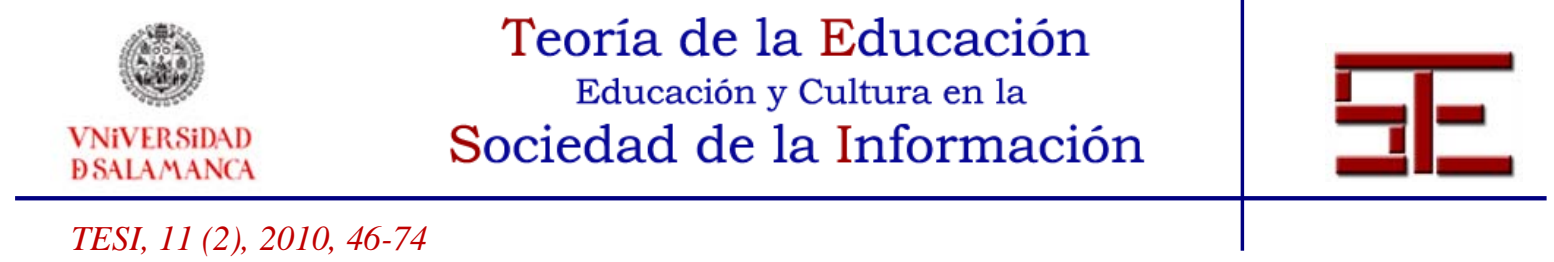

Física Médica. En cualquier caso, concluye en la necesidad de disponer de una lista exhaustiva de estos instrumentos, lo que sería de gran valor para la educación y la formación en nuestra disciplina.

Dikshit et al. (2005) desarrollaron un sistema de simulación de imágenes médicas para la enseñanza de los estudiantes, así como de profesionales que pueden trabajar en este campo. La herramienta proporciona un completo tutorial de simulaciones que introduce al usuario en los fundamentos de diferentes técnicas de imagen como radiografías, tomografía computarizada, resonancia magnética, ecografía o tomografía por emisión de positrones. Aunque existen numerosas páginas web que ofrecen documentación online sobre imagen radiológica, hay muy pocas que proporcionen simulaciones computacionales interactivas. En este sentido, la plataforma ofrece a los alumnos una visión de los principios físicos y técnicos de la imagen en radiología en un laboratorio virtual interactivo.

En la Facultad de Medicina de la Universidad de Salamanca, la Unidad de Física Médica del Área de Radiología y Medicina Física trabaja, desde hace tiempo, en la implementación de contenidos educativos en la plataforma informática FISIMED, el Sistema de Gestión de Contenidos (CMS) de apoyo al proceso de enseñanzaaprendizaje online de nuestra disciplina al que hicimos referencia en la introducción de este trabajo. En la actualidad, como comentamos en los objetivos del mismo, nuestro esfuerzo se dirige especialmente al diseño y desarrollo de animaciones y simulaciones computacionales.

A modo de ejemplo, nos referiremos a una simulación que intenta "hacer visible" al alumno el fenómeno de resonancia frente al de relajación, claves para la comprensión de los fundamentos físicos de la resonancia magnética. Asimismo, una animación en la que es estudiante puede "visualizar" el efecto piezoeléctrico y el efecto piezoeléctrico inverso, esto es, la base del método utilizado en medicina para la producción de ultrasonidos de frecuencias elevadas, aquellas que se emplean en ultrasonografía.

Uno de los avances tecnológicos más importantes alcanzados en el campo de la radiología digital ha sido la consecución de imágenes basadas en la resonancia magnética. La resonancia magnética emplea campos magnéticos y radiación no ionizante de radiofrecuencia del espectro electromagnético en la obtención de dichas imágenes.

"Los fundamentos físicos de la técnica se resumen en los puntos siguientes:

- El momento magnético de los núcleos de hidrógeno del organismo ha de ser orientado en la dirección de un campo magnético constante, homogéneo y de gran intensidad $(0,5-3 \mathrm{~T})$.

- Un pulso de ondas electromagnéticas (ondas de radiofrecuencia, RF) de una frecuencia precisa, igual a la frecuencia de resonancia de los núcleos de hidrógeno, hace que el momento magnético

Francisco Javier Cabrero Fraile, José Miguel Sánchez Llorente,

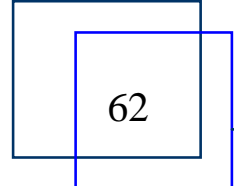
Ana B. Sánchez García, Javier Borrajo Sánchez, $M^{a}$ José Rodríguez Conde, Marta Cabrero Hernández y Juan Antonio Juanes Méndez

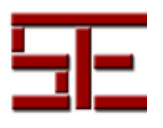




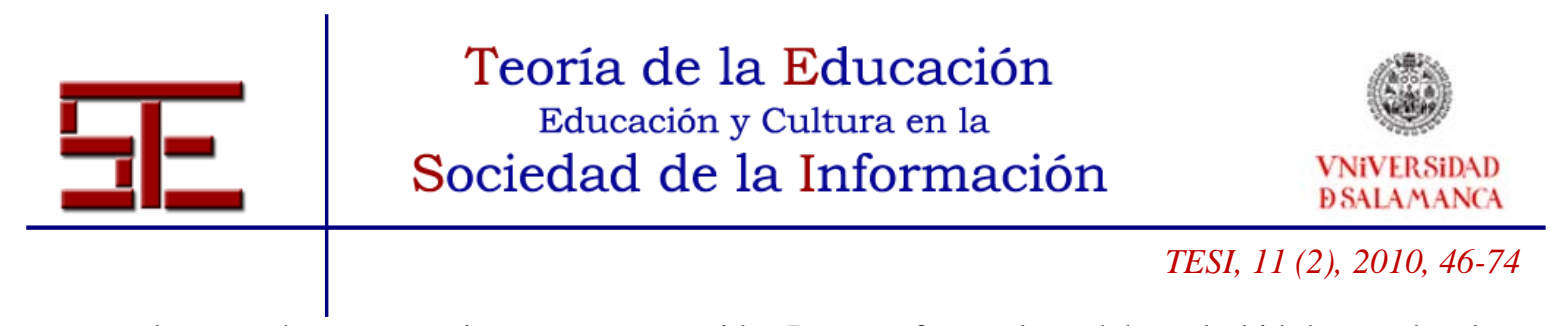

de estos átomos se oriente en otro sentido. De esta forma, los núcleos de hidrógeno absorben energía del haz de radiofrecuencia y empiezan a girar acompasados (resonancia).

- Cuando cesa el pulso de RF se produce una liberación de energía, también en forma de onda de radiofrecuencia, como resultado de la vuelta del momento magnético de los núcleos a su orientación inicial (relajación).

- La señal de relajación (energía liberada), portadora de gran cantidad de información, es detectada con una antena y analizada.

- Las señales provenientes de cada volumen elemental de la zona explorada son sometidas a un tratamiento informático que proporciona una imagen de dicha zona” (Cabrero, 2004, 166).

Los fenómenos de resonancia y relajación se representan mediante vectores de magnetización, longitudinal (eje z) y transversal (plano x-y), de manera que: “el vector de magnetización $\mathrm{M}$ describe durante la relajación una espiral ascendente, una superficie en
forma de pabellón de trompeta que es el resultado de los fenómenos de relajación longitudinal y
transversal que ocurren simultáneamente. Sin embargo, durante la excitación, el vector de
magnetización describe una espiral formando una superficie en forma de esfera debido a la reducción
progresiva de su componente longitudinal y a la aparición de un componente transversal” (fig. 1)
(Cabrero, 2004, 177).
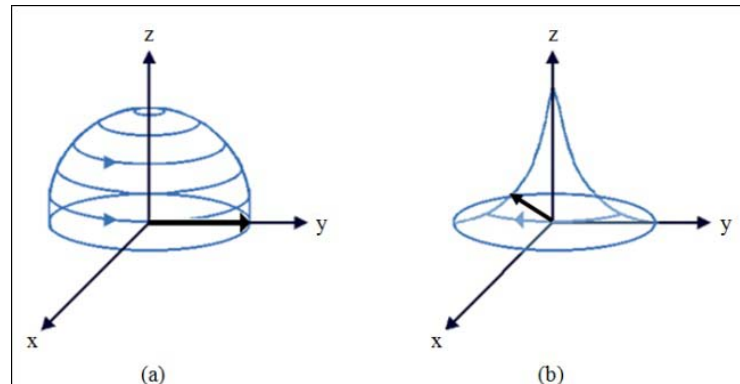

Figura 1. Resonancia (a) frente a relajación (b) (Tomado de Cabrero, 2004).

La simulación fue realizada en "Adobe Shockwave” (generalmente, estas simulaciones se programan bajo Macromedia Flash). Las versiones 4 y posteriores de Flash incorporan el lenguaje de programación orientado a objetos ActionScript, un lenguaje muy similar al JavaScript basado en la especificación estándar de industria ECMA-262. ActionScript, cuya misión dentro de Flash es animar aplicaciones, permite la conexión con otros interfaces así como la inclusión de herramientas adicionales como parte del código. Así, en la simulación de la variación en el vector magnetización, se ha contado con la facilidad de ActionScript para incorporar las trayectorias descritas por dicho vector, las cuales han sido generadas en el programa Mathematica (Wolfram Research: sistema de álgebra computacional) a partir de las ecuaciones preestablecidas. El usuario puede elegir, mediante listas desplegables, el tipo de tejido y núcleo sobre el que se va a experimentar el fenómeno de resonancia magnética representando, de este modo, el proceso físico que tiene lugar en la unidad de diagnóstico por imagen. La figura 2

Francisco Javier Cabrero Fraile, José Miguel Sánchez Llorente, \begin{tabular}{ll|l|}
\cline { 3 - 4 } & \\
\hline & $\begin{array}{c}\text { Ana B. Sánchez García, Javier Borrajo Sánchez, Ma José Rodríguez } \\
\text { Conde, Marta Cabrero Hernández y Juan Antonio Juanes Méndez }\end{array}$ & 63 \\
\hline
\end{tabular} 


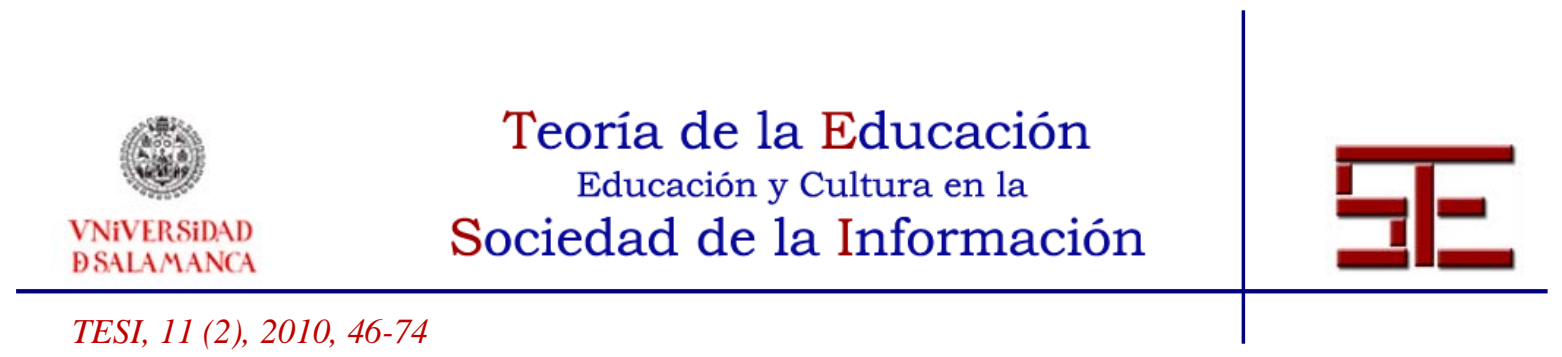

muestra la formación de la superficie en forma de esfera que describe el vector de magnetización durante la excitación.

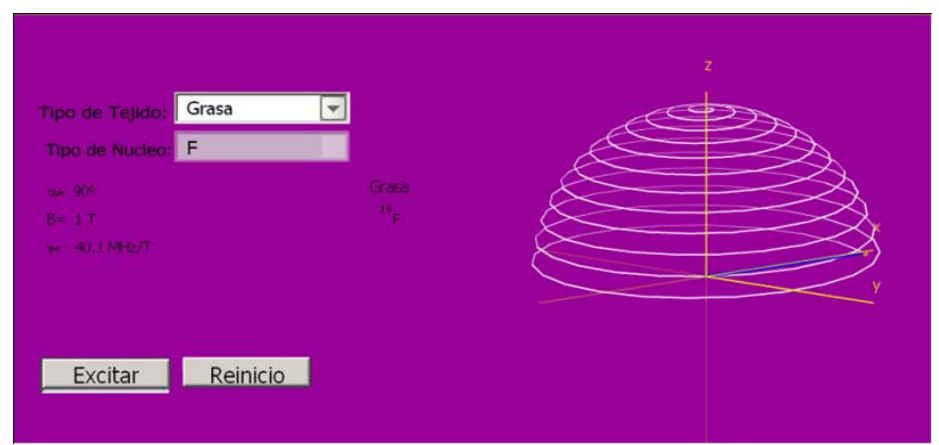

Figura 2. FISIMED: Simulación interactiva (fenómeno de resonancia magnética).

Por otra parte, una de las técnicas de diagnóstico por imagen más utilizadas en medicina es la ecografía, término que hace referencia a la visualización de imágenes del organismo mediante la utilización de ultrasonidos. En la práctica, como señalamos anteriormente, el método utilizado para la producción de ultrasonidos de frecuencias elevadas, como las utilizadas en ecografía, se basa en el efecto piezoeléctrico descubierto por los hermanos Curie en el año 1880. El fenómeno físico de la piezoelectricidad es imposible de visualizar por nuestros alumnos en el laboratorio. No obstante, puede ser presentado en forma de animación para conseguir una mejor comprensión del mismo.

\begin{abstract}
“El efecto piezoeléctrico consiste en que al ejercer una presión sobre determinados cristales tallados (láminas piezoeléctricas) aparecen cargas eléctricas de signo opuesto en sus superficies. Así, una de ellas queda cargada positivamente y la otra negativamente. Ahora bien, si la compresión se convierte en tracción, también aparecen cargas eléctricas superficiales pero la polaridad es opuesta a la anterior, es decir, se invierte el signo de las cargas. Recíprocamente, al aplicar a este tipo de cristales una tensión eléctrica alterna que cambia de forma periódica el sentido de las cargas, se producen dilataciones y compresiones del cristal (sus moléculas se reordenan y se produce una deformidad mecánica), esto es, vibraciones mecánicas ultrasonoras. Esta reversibilidad del efecto piezoeléctrico recibe el nombre de efecto piezoeléctrico inverso.” (Cabrero, 2004, 201).
\end{abstract}

En la animación se reproducen ambos efectos, aunque es el efecto piezoeléctrico inverso el mecanismo responsable de la producción de los ultrasonidos que se emplean en el ámbito médico (figs. 3-5).

Francisco Javier Cabrero Fraile, José Miguel Sánchez Llorente, Ana B. Sánchez García, Javier Borrajo Sánchez, $M^{a}$ José Rodríguez Conde, Marta Cabrero Hernández y Juan Antonio Juanes Méndez 


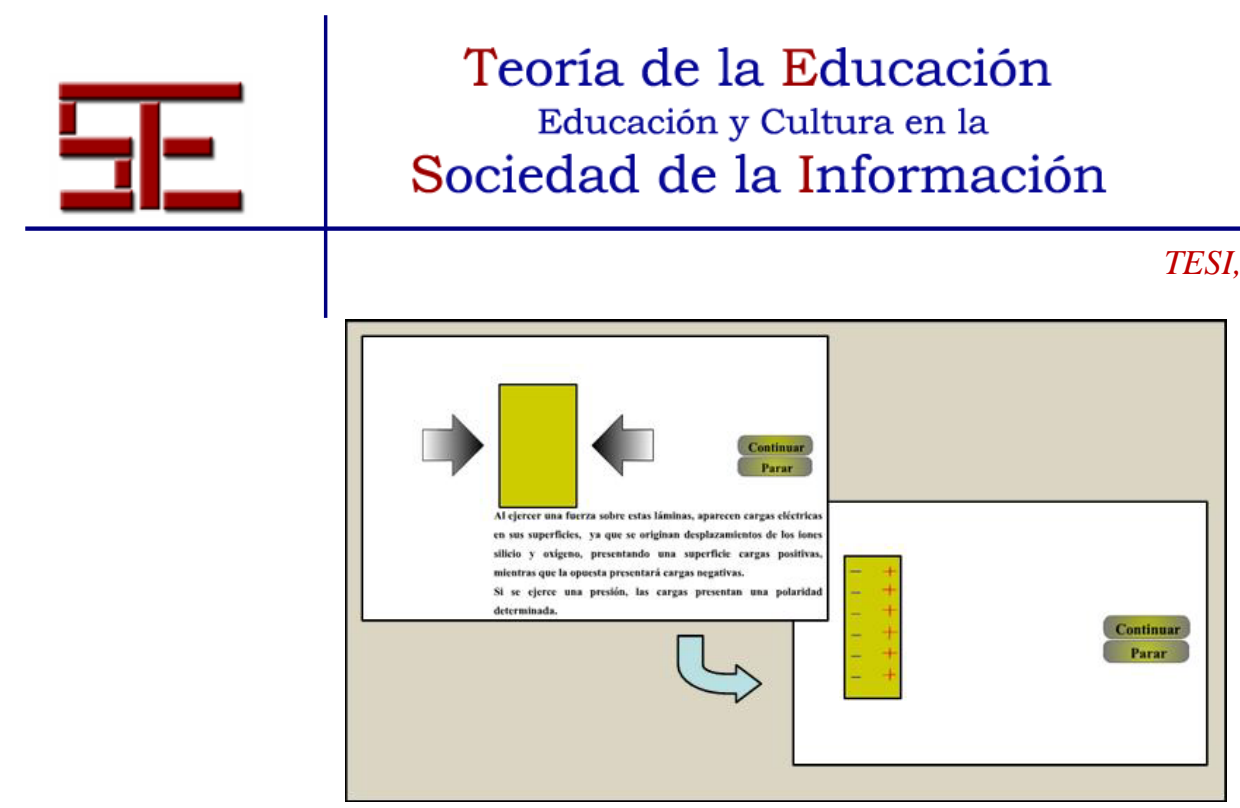

Figura 3. Efecto piezoeléctrico: al ejercer una presión sobre una lámina piezoeléctrica, aparecen cargas eléctricas de signo opuesto en sus superficies.

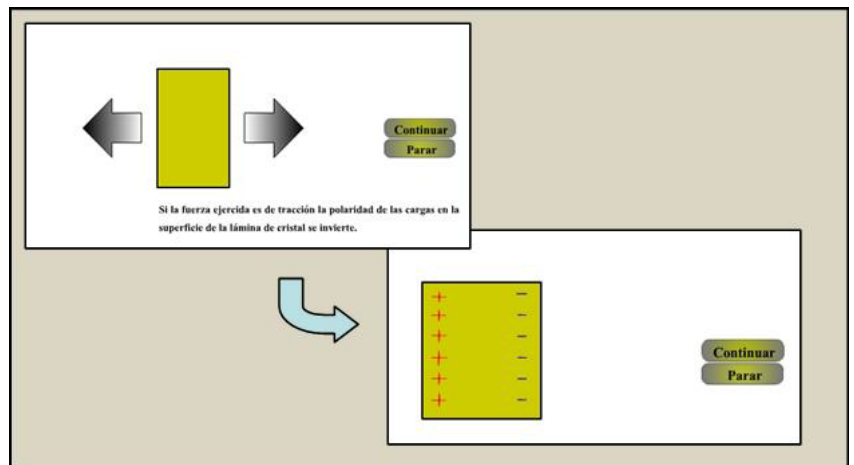

Figura 4. Efecto piezoeléctrico: si la compresión se convierte en tracción, se invierte el signo de las cargas.

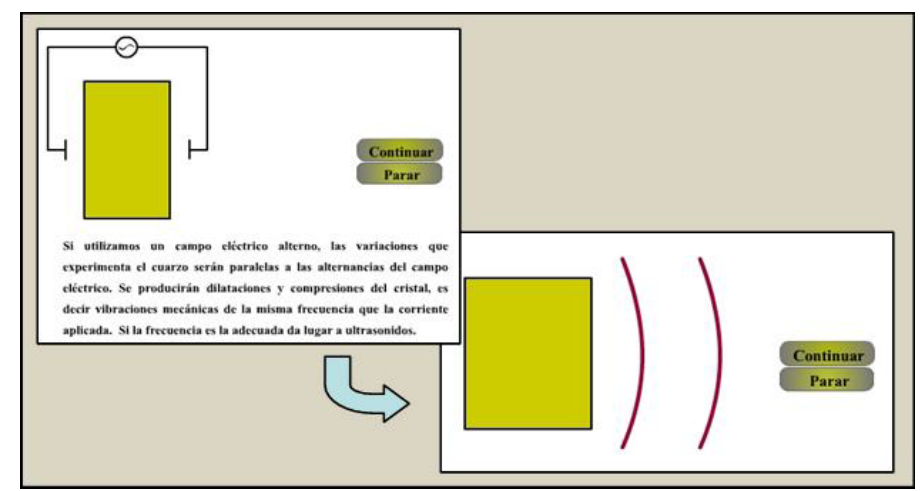

Figura 5. Efecto piezoeléctrico inverso: al aplicar una tensión eléctrica alterna se producen vibraciones mecánicas ultrasonoras.

Los ejemplos anteriores constituyen una muestra representativa del papel que juegan las simulaciones computacionales en el proceso de enseñanza-aprendizaje de la Física

Francisco Javier Cabrero Fraile, José Miguel Sánchez Llorente,

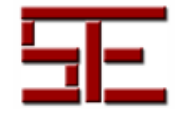

Ana B. Sánchez García, Javier Borrajo Sánchez, $M^{a}$ José Rodríguez Conde, Marta Cabrero Hernández y Juan Antonio Juanes Méndez 


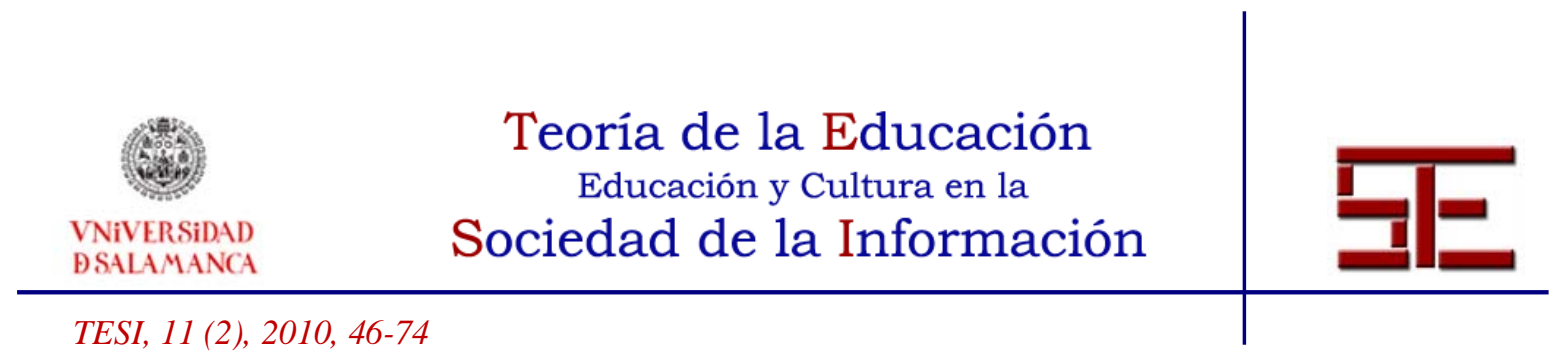

Médica, aunque se refieren a aspectos concretos de la misma como, por ejemplo, los relacionados con la imagen médica. Sin embargo, de acuerdo con las consideraciones introductorias sobre nuestra disciplina, el campo de actuación de la Física Médica es muy amplio, haciéndose evidente la necesidad de un conocimiento general de la Física que proporcione una base sólida para la comprensión de sus fundamentos. En este sentido, en relación con el uso de simulaciones informáticas en la docencia de la materia, se hace imprescindible disponer de una colección lo más completa posible en temas de Física General afines a la misma.

\section{3.- Simulaciones computacionales en la enseñanza de la Física en general.}

En un reciente estudio, Zavala y Velarde (2009) plantearon la siguiente pregunta “¿tiene el uso de simulaciones computacionales con una estrategia educacional activa resultados similares de aprendizaje al uso de equipo real con la misma estrategia educacional activa?’. Los resultados de la investigación mostraron que las simulaciones pueden ser tan positivas como el uso del equipo real de laboratorio, incluso pueden llegar a ser más efectivas para la comprensión de ciertos conceptos (en este estudio, sobre circuitos eléctricos). No obstante, la experiencia adquirida por el alumno al trabajar con el equipo del laboratorio se traduce en un mayor conocimiento de cómo funcionan realmente los elementos del circuito. La experiencia fue realizada con la simulación Circuit Construction Kit PhET, un proyecto online de la Universidad de Colorado (USA).

Aunque existen muchos tipos de simulaciones computacionales, la Universidad de Colorado ha desarrollado una gran colección de animaciones y simulaciones en diversos campos y, entre ellos, la Física, basadas en Java pero con el apoyo de Flash para su diseño. En definitiva, las simulaciones interactivas PhET, disponibles libremente en la web $\mathrm{PhET}$, son el resultado de un extraordinario esfuerzo para intentar ayudar a los estudiantes en el proceso de enseñanza-aprendizaje de las ciencias. Constituyen un conjunto de herramientas interactivas, ampliamente probadas y evaluadas para su validación, que permiten a los usuarios establecer conexiones entre los fenómenos reales y la ciencia subyacente que los explica (fig. 6).

(Disponible en: http://phet.colorado.edu/simulations/index.php?cat=Featured_Sims).

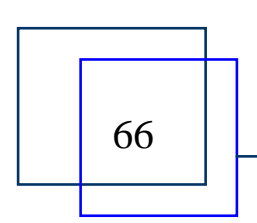

Francisco Javier Cabrero Fraile, José Miguel Sánchez Llorente, Ana B. Sánchez García, Javier Borrajo Sánchez, $M^{a}$ José Rodríguez Conde, Marta Cabrero Hernández y Juan Antonio Juanes Méndez 


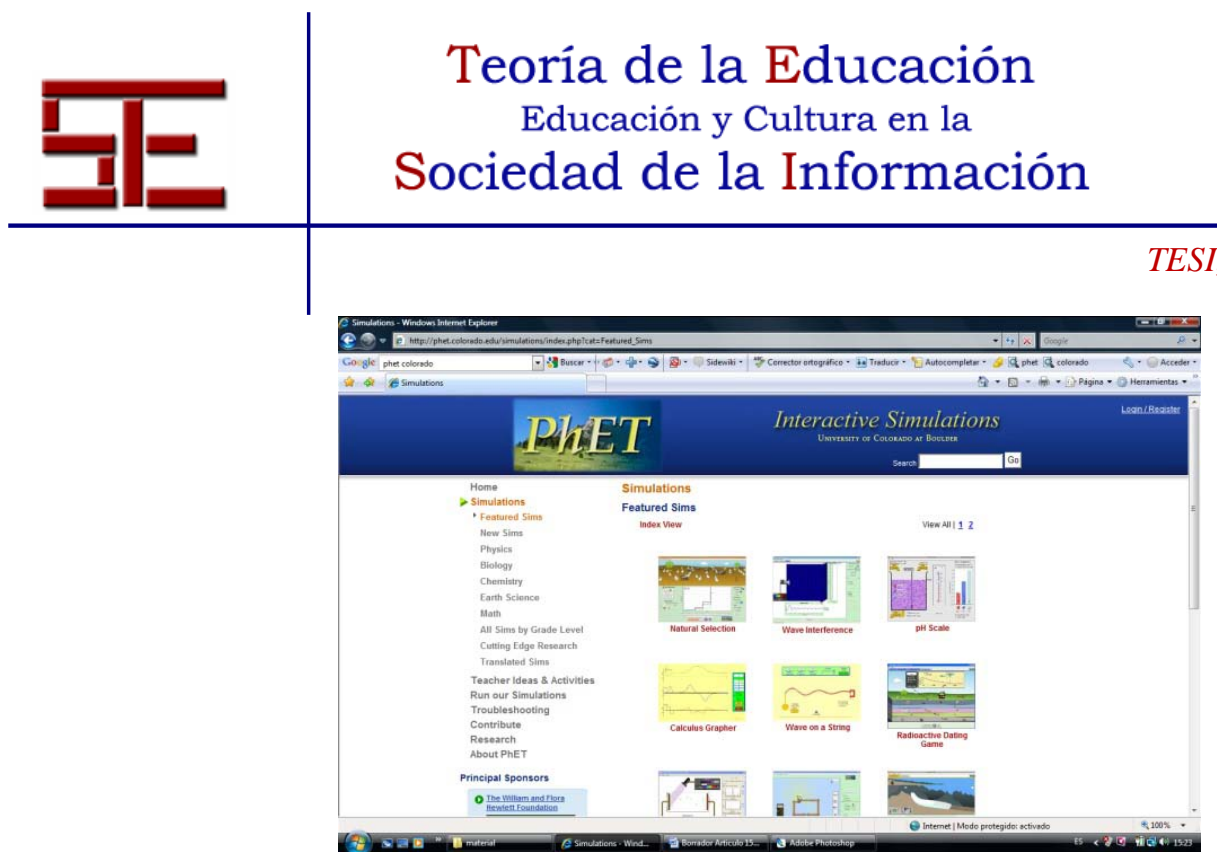

Figura 6. Web PhET Simulaciones interactivas de la Universidad de Colorado (USA).

La figura 7 muestra una de las pantallas de la simulación sobre resonancia magnética de cabeza, un ejemplo de cómo PhET ofrece a los estudiantes de Física Médica, simulaciones que, sin lugar a dudas, pueden ayudar a los alumnos a comprender mejor los principios físicos y los recursos técnicos en los que se basan muchas de las técnicas que se emplean en Medicina.

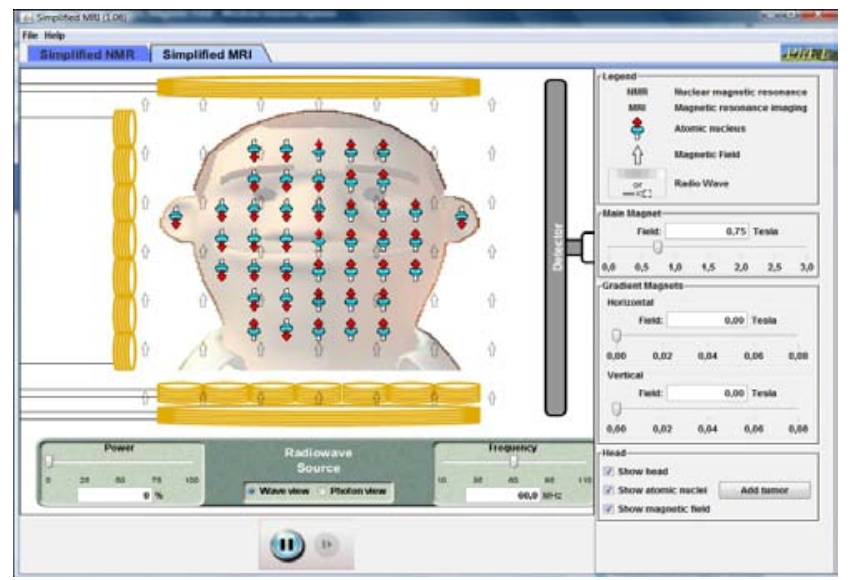

Figura 7. PhET (simulación interactiva sobre resonancia magnética).

Existen otras muchas páginas web que aparecen en la red como laboratorios virtuales. Por ejemplo, un laboratorio con simulaciones Java sobre oscilaciones y ondas, óptica, etc. disponible en http://www.enciga.org/taylor/lv.htm; o el laboratorio virtual de Física N.T.N.U. (NTNUJAVA Virtual Physics Laboratory), probablemente la mayor colección de applets de Física que hay en la red (disponible en: http://www.phy.ntnu.edu.tw/ntnujava/).

Francisco Javier Cabrero Fraile, José Miguel Sánchez Llorente, \begin{tabular}{ll|l|}
$\begin{array}{c}\text { Ana B. Sánchez García, Javier Borrajo Sánchez, Ma José Rodríguez } \\
\text { Conde, Marta Cabrero Hernández y Juan Antonio Juanes Méndez }\end{array}$ & 67 \\
\hline
\end{tabular} 


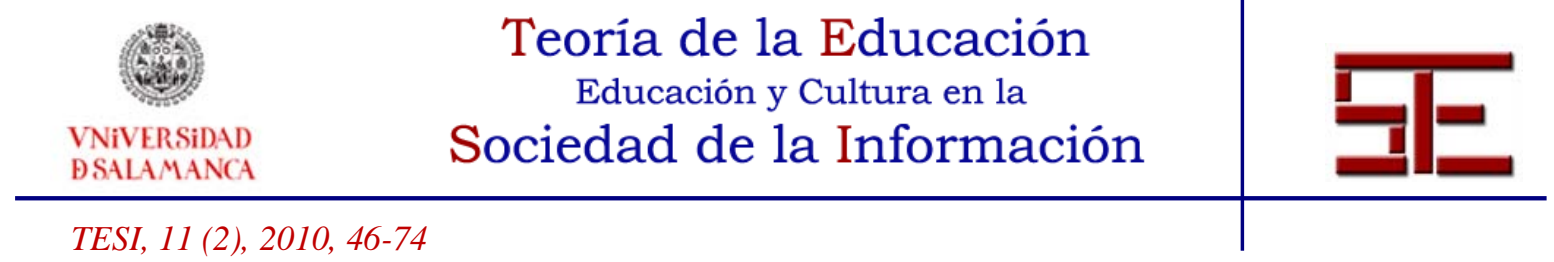

MERLOT (Multimedia Educational Resource for Learning and Online Teaching) es una web con software de aprendizaje sobre distintas disciplinas. En MERLOT Physics se dispone de una amplia colección de materiales sobre las distintas ramas de la Física con multitud de animaciones y simulaciones.

(Disponible en: http://www.merlot.org/merlot/materials.htm?category=2736)

Teachers'domain, al igual que la anterior, ofrece material didáctico, entre otros, sobre Ciencia y, en concreto sobre Ciencias Físicas. Proporciona una amplia colección de vídeos y simulaciones descargables previo registro.

(Disponible en: http://www.teachersdomain.org/collection/k12/sci.phys/)

SMETE Digital Library es una biblioteca online y un portal de servicios para estudiantes y profesores. Desde esta web se puede acceder a una gran cantidad de material didáctico y pedagógico, incluidas simulaciones computacionales en Física.

(Disponible en: http://www.smete.org/smete/)

http://www.ite.educacion.es/ es la página principal del Instituto de Tecnologías Educativas, la unidad del Ministerio de Educación responsable de la integración de las TIC en las etapas educativas no universitarias. Ofrece gran cantidad de recursos educativos, muchos de ellos animaciones y/o simulaciones interactivas, relacionados con distintas asignaturas como, por ejemplo, Física y Química (fig. 8).

(Disponible en: http://www.isftic.mepsyd.es/profesores/asignaturas/fisica_y_quimica/)

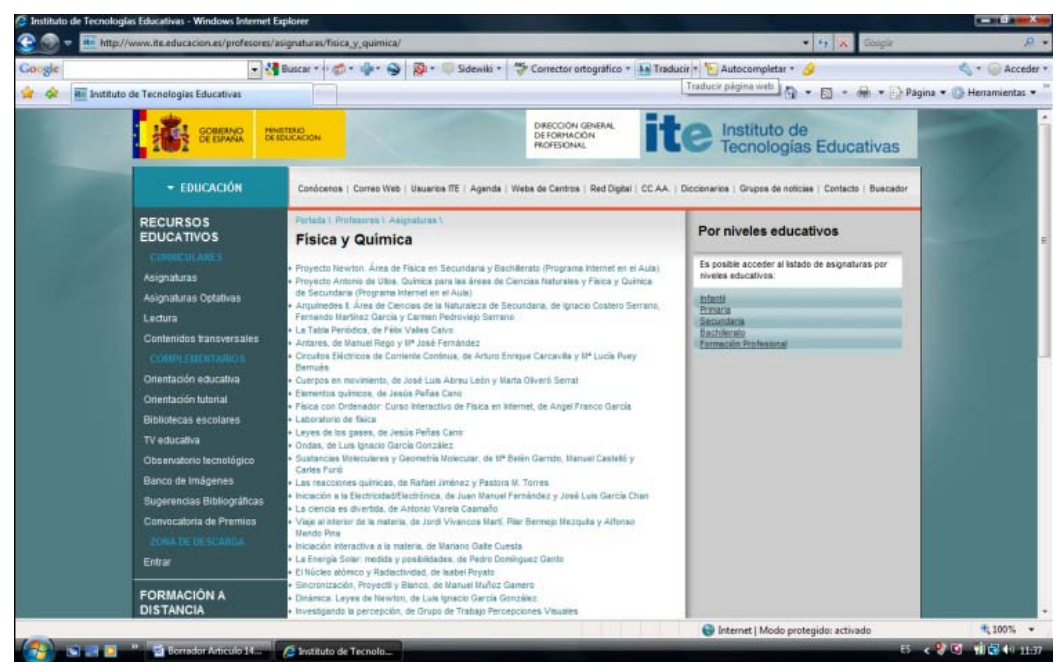

Figura 8. Recursos Educativos de Física y Química (Ite).

Francisco Javier Cabrero Fraile, José Miguel Sánchez Llorente, Ana B. Sánchez García, Javier Borrajo Sánchez, $M^{a}$ José Rodríguez Conde, Marta Cabrero Hernández y Juan Antonio Juanes Méndez 


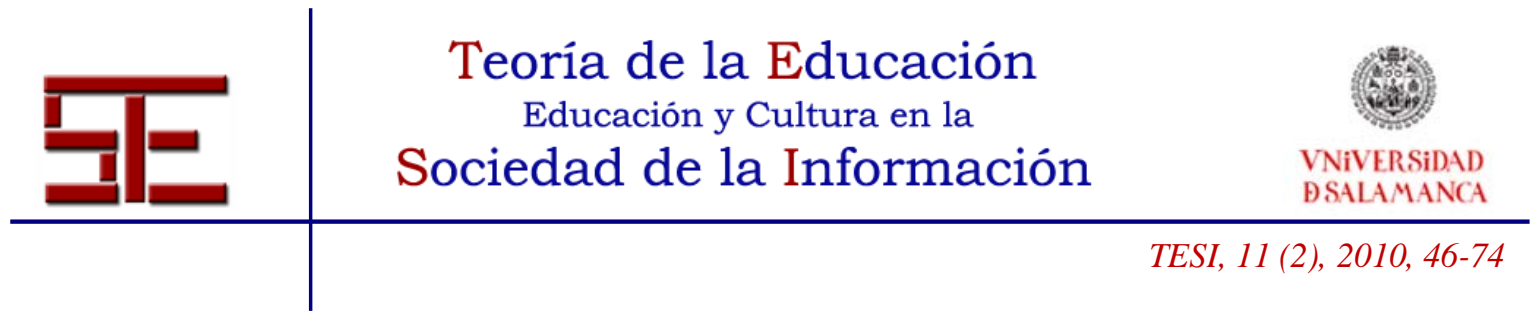

El Curso Interactivo de Física en Internet de A. Franco García, cuya última actualización corresponde al mes de noviembre de 2009, "es un curso de Física general que trata desde conceptos simples como el movimiento rectilíneo hasta otros más complejos como las bandas de energía de los sólidos. La interactividad se logra mediante los 646 applets insertados en sus páginas webs que son simulaciones de sistemas físicos, prácticas de laboratorio, experiencias de gran relevancia histórica, problemas interactivos, problemas-juego, etc.” (fig. 9).

(Disponible en: http://www.sc.ehu.es/sbweb/fisica_/)

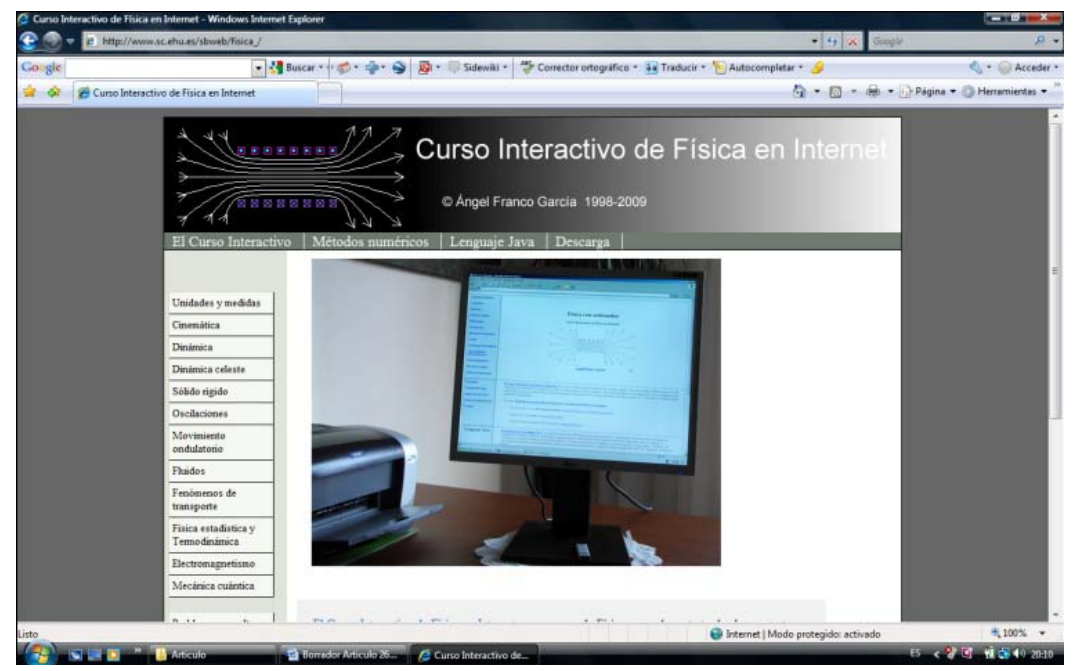

Figura 9. Página principal del Curso Interactivo de Física de A. Franco García.

Physics 2000 constituye un viaje interactivo a través de la Física Moderna, apoyado firmemente en el uso interactivo de applets. Entre ellos, los que forman parte del apartado "Einstein's Legacy” proporcionan una visión simulada de fenómenos físicos y/o principios físicos que pertenecen al campo de estudio de la Física Médica (ondas electromagnéticas, rayos $\mathrm{X}$, mecanismos de producción de rayos $\mathrm{X}$, fundamentos de la tomografía computarizada, microondas o láser, entre otros).

(Disponible en: http://www.colorado.edu./physics/2000/index.pl).

Finalmente, aunque lo más habitual es descubrir en la red páginas con simulaciones Java (applets), existen otras en las que encontramos animaciones basadas en Flash de Macromedia. Un ejemplo es la web del profesor Harrison de la Universidad de Toronto, de la que existe una traducción autorizada disponible en http://www.meetphysics.net/David-Harrison/index_spa.html.

En definitiva, una gran colección de contenidos educativos distribuidos a través de Internet, cuya incorporación a la docencia puede generar, como señalan Ruzo y Rodeiro

Francisco Javier Cabrero Fraile, José Miguel Sánchez Llorente, Ana B. Sánchez García, Javier Borrajo Sánchez, Ma José Rodríguez
Conde, Marta Cabrero Hernández y Juan Antonio Juanes Méndez 


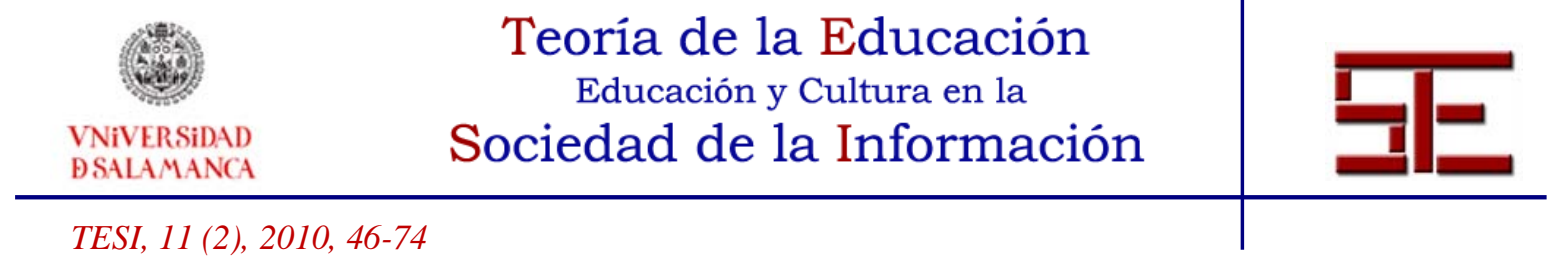

(2006) en relación con la incorporación de las TIC a la docencia en las aulas, una actitud activa y un interés hacia su utilización en las actividades asociadas al proceso de aprendizaje de los alumnos.

\section{6.- CONCLUSIONES}

El desarrollo del Espacio Europeo de Educación Superior y el uso de las Tecnologías de la Información y la Comunicación va a favorecer un cambio en la práctica pedagógica del docente. Las TIC proporcionarán recursos que permitirán a las universidades mejorar la calidad de la enseñanza. La extensión de la formación a distancia, sobre todo por la implantación de plataformas virtuales, es una realidad en las instituciones universitarias. La plataforma informática FISIMED (LMS) de la Unidad de Física Médica de la Facultad de Medicina de la Universidad de Salamanca permite la enseñanza y aprendizaje a distancia basada en las TIC. La combinación de esta modalidad, con estrategias docentes de tipo tradicional, parece muy adecuada en el ámbito de la educación médica.

En relación con los contenidos implementados en nuestra plataforma, y en el campo de la Física Médica (o de la Física, en general), existe una tendencia a desarrollar materiales basados en animaciones o simulaciones informáticas de determinados procesos. La educación médica basada en simulaciones se presenta como una herramienta de gran valor en la formación de pregrado de los futuros profesionales, tanto en las disciplinas clínicas como preclínicas. No obstante, refiriéndonos en concreto al campo de las simulaciones computacionales, la falta de apoyo y de reconocimiento que tiene el esfuerzo realizado por los profesores, se presenta como una barrera importante para el desarrollo e implementación de materiales educativos.

La utilización de simulaciones informáticas en el campo de la Física, permite a los alumnos disponer de una ayuda para la comprensión de conceptos que constituyen una tarea difícil para ellos y que no pueden ser "visualizados" en el aula o en el laboratorio. Por otra parte, el empleo de estos materiales consigue aumentar la motivación de los estudiantes como ocurre, en general, con el uso de sistemas alternativos de enseñanza basados en las nuevas tecnologías.

En la actualidad, el software disponible para el desarrollo de animaciones y/o simulaciones ha evolucionado enormemente, con incorporación de nuevas características que permiten mejorar la calidad de las aplicaciones realizadas frente a las tradicionales programadas en Java o Adobe Shockwave. La tendencia hacia el empleo de software de renderizado 3D, como Maya, 3D Studio Max, Softimage, Lightwave o Cinema 4D, entre otros, que incluyen herramientas de tratamiento de elementos

Francisco Javier Cabrero Fraile, José Miguel Sánchez Llorente,

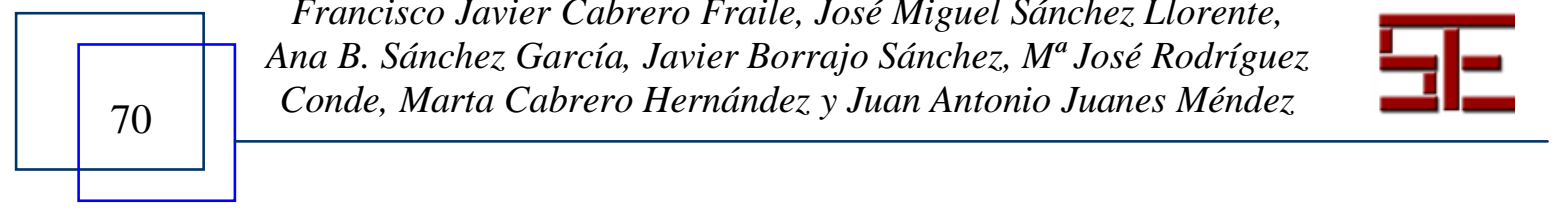




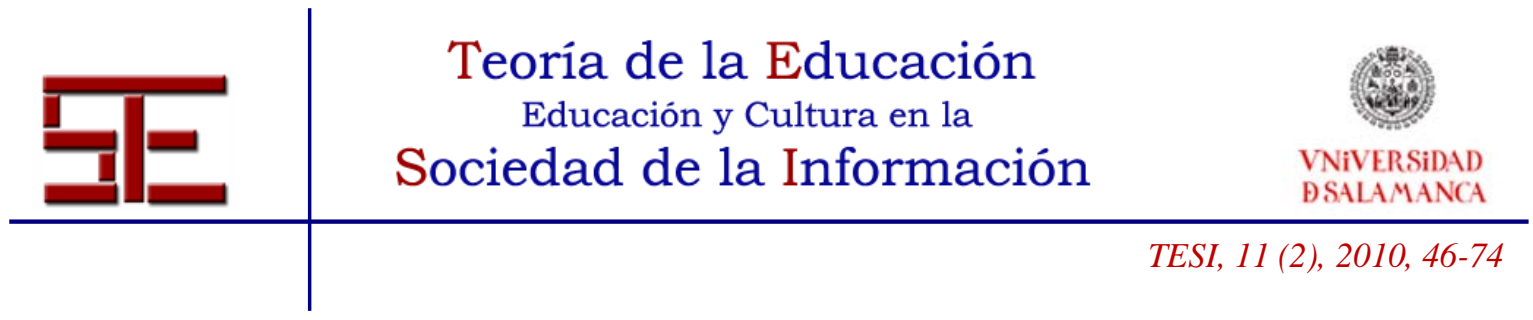

orgánicos (personas, etc.), plugins y mejoras técnicas de modelado, iluminación y texturizado, se muestra como el presente y futuro de la animación y simulación informática en el campo de las Ciencias de la Salud.

Finalmente, la aplicación de las nuevas tecnologías a los procesos de enseñanza y aprendizaje de las Ciencias de la Salud hace que converjan distintos sectores profesionales (médicos, odontólogos, físicos, pedagogos, informáticos,...) en un proceso de investigación interdisciplinar que aporta un valor añadido a los trabajos, ya que puede estimular la innovación e investigación y contribuir a la mejora de la calidad de la enseñanza.

\section{7.- BIBLIOGRAFÍA}

Blanco, C. Y Vázquez, G. (2006). La Fundación IAVANTE, y su apuesta por el e.Learning. Educación Médica, 9 (Supl. 2), 5.

Bonete, R. (2006). La adaptación de la Universidad de Salamanca al Espacio Europeo de Educación Superior: un desafío para todos. Salamanca, Universidad de Salamanca, Vicerrectorado de Planificación e Innovación Docente. Disponible en: http://campus.usal.es/ ofeees/documentos_interes_generales.htm

Brusilovsky, P. (1994). The Construction and Application of Student Models in Intelligent Tutoring Systems. Journal of Computer and Systems Sciences International, 32 (1), 70- 89.

Cabero, J. (Director) (2005). Formación del profesorado universitario en estrategias metodológicas para la incorporación del aprendizaje en red en el Espacio de Educación Superior (EEES). Disponible en: http://campus.usal.es/ ofeees/estudios_informes.htm

Cabrero, F.J. (2004). Imagen radiológica. Principios físicos e instrumentación. Barcelona, Masson, S.A.

Cabrero, F.J., Sánchez, J.M., Rodríguez, M.J., Borrajo, J., Aparicio, M., Martín, A., Gómez, P. Y Gutiérrez, M.J. (2009). FISIMED: Contenidos implementados en el aprendizaje “on-line” de la Física Médica en el marco del Espacio Europeo de Educación Superior. Alicante: XVII Congreso Nacional de SEFM y XII Congreso Nacional de SEPR (trabajo completo en edición (CD) y en web).

Cabrero, F.J., Rodríguez Conde, M.J., Romero, J., Juanes, J.A., Asensio, M. Y Cabrero, A. (2008). Design and experimental validation of a multimedia program in oral radiology, en Riko Koboyashi (ed.). New Educational Technology. New York: Nova Science Publishers, Inc., 213-227.

Comisión Para La Renovación De Las Metodologías Educativas En La Universidad (2006). Propuesta para la renovación de metodologías educativas en la

Francisco Javier Cabrero Fraile, José Miguel Sánchez Llorente,

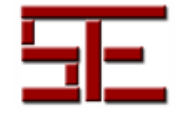
Ana B. Sánchez García, Javier Borrajo Sánchez, Ma José Rodríguez Conde, Marta Cabrero Hernández y Juan Antonio Juanes Méndez 


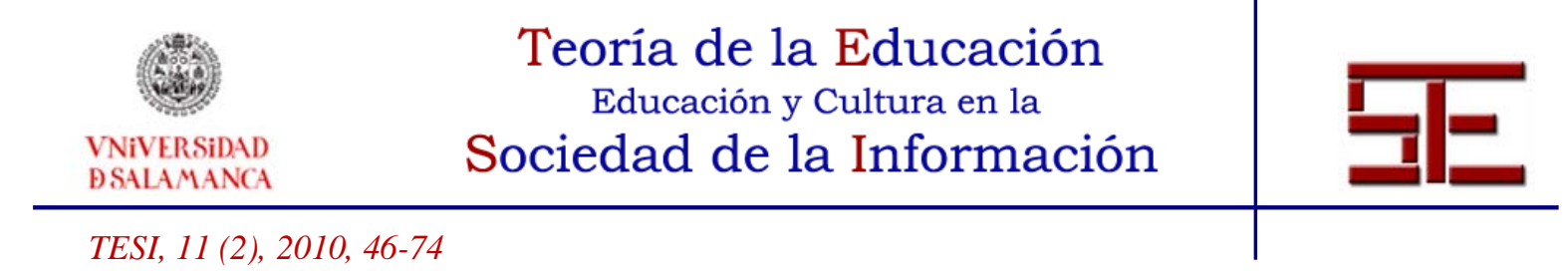

Universidad. Ministerio de Educación y Ciencia. Ministerio de Educación y Ciencia. Secretaría de Estado de Universidades e Investigación.

Conferencia De Rectores De Las Universidades Españolas (2006). Las TIC en el Sistema Universitario Español (2006): Un análisis estratégico. Dres.: Barro Ameneiro, S. y Burillo López, P. Madrid: Conferencia de Rectores de las Universidades Españolas. Disponible en: http://campus.usal.es/ ofeees/estudios_informes.htm

Demir, S.S. (2005). Simulation-based training in Electrophysiology by iCell: Engineering in Medicine and Biology Society. IEEE-EMBS 2005. 27th Annual International Conference of the IEEE, 851-854.

Demir, S.S. Y Velipasaoglu, E.O. (2003). Simulation-based education and training resource: iCell, en Information Technology Applications in Biomedicine, 2003. 4th International IEEE EMBS Special Topic Conference on Information Technology Applications in Biomedicine, 24 - 26 April 2003, 106-109.

Duart, J. M. (2006). Estrategias en la introducción y uso del e.Learning en educación superior. Educación Médica, 9 (supl. 2), 16-22.

Fernández, S., Rodeiro, D. Y Ruzo, E. (2006). Las TIC en la Educación Superior: una visión general, en Las TIC en el Sistema Universitario Español (2006): Un análisis estratégico. Dres.: Barro Ameneiro, S. y Burillo López, P. Madrid: Conferencia de Rectores de las Universidades Españolas.

Fundación Iavante (2006). Innovación docente vía TIC. Educación Médica, 9 (supl. 2).

Galindo, J. Y Visbal, S. (2007). Simulación, herramienta para la educación médica. Salud Uninorte. Barranquilla (Col.), 23, 79-95.

Grupo De Promotores De Bolonia En España (Programa Sócrates 2005 - 1678/001 SO2-21BOPR): Preguntas frecuentes sobre: la estructura de las enseñanzas universitarias, el Grado y el Posgrado.

Holmes, B. Y Gardner, J. (2006). e.Learning. Concepts and Practice. London, SAGE Publications Ltd.

Lago, J. (2006). Situación actual de estándares e.Learning y aplicación en entornos de Software Libre. Educación Médica, 9 (Supl. 2): 28-33.

Marqués, P., Dorado, C., Bosco, A. Y Santiveri, N. (2006). Las TICs como instrumento de apoyo a las actividades de los docentes universitarios y de sus alumnos en el marco de la implantación de los créditos ECTS. Las claves del éxito. Disponible en: http://campus.usal.es/ ofeees/formacion_eees.htm

Mcdermott, L.C. Y Redish, E.F. (1999). Resource Letter: PER-1: Physics Education Research. American Journal of Physics, 67: 755.

Monsky, W.L., Levine, D., Mehta T.S., Kane, R.A., Ziv, A., Kennedy, B. Y Nisnbaum, H. (2002). Using a sonographic simulator to assess residents before overnight call. AJR, 178: 35-39.

Francisco Javier Cabrero Fraile, José Miguel Sánchez Llorente,

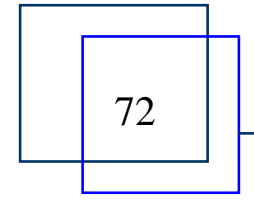
Ana B. Sánchez García, Javier Borrajo Sánchez, $M^{a}$ José Rodríguez Conde, Marta Cabrero Hernández y Juan Antonio Juanes Méndez

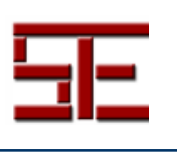




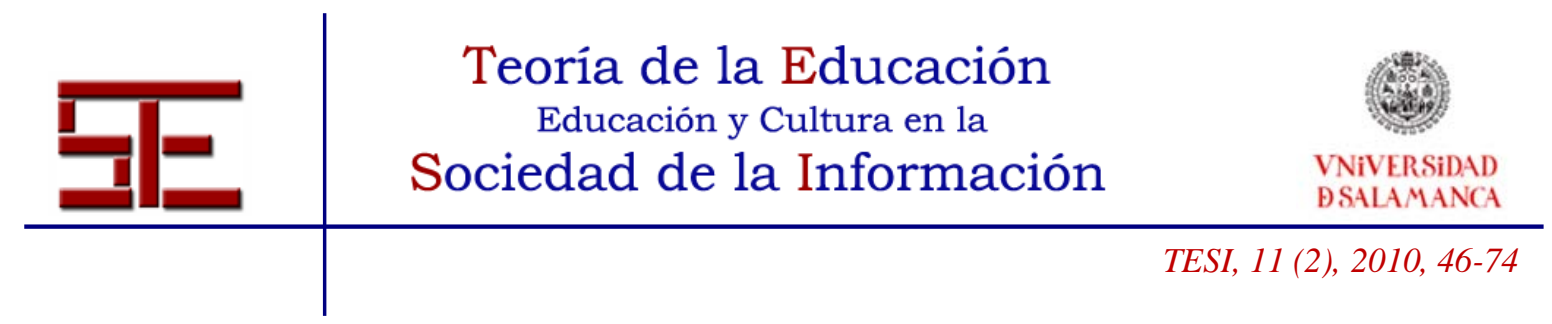

Ortega, J., Rodríguez Conde, M.J., López, R., Ortega, J. Y Sánchez, A.B. (2002). La "nueva" formación del profesorado universitario y el entorno de la Universidad de Salamanca para la docencia en red (EUDORED-Jle/Edustance). II Congreso Internacional “Docencia Universitaria e Innovación”. Tarragona 1, 2 y 3 de julio de 2002.

Physics Education Technology Project At The University Of Colorado (2007). Disponible en: http://phet.colorado.edu/simulations

Pls Rambol Management (2004): Studies in the Context of the E-Learning Initiative: Virtuals Models of European Universities (Lot 1). Draft Final Report to the UE25 Commission, DG Education \& Culture. Disponible en: http://www.elearningeuropa.info/extras/pdf/virtual_models.pdf

REAL DECRETO 1393/2007, de 29 de octubre, por el que se establece la ordenación de las enseñanzas universitarias oficiales. B.O.E. 30 de octubre de 2007. Disponible en: http://campus.usal.es/ ofeees/

Riley, D., Otamendi, A. Y Álvarez, J. (2006). La combinación del e.Learning con otras estrategias docentes. Educación Médica, 9 (Supl. 2): 51-57.

Rojas, J.F. Y Morales, M.A. (2009). Física computacional: una propuesta educativa. Rev Mex Fis, 55: 97-111.

Ruzo, E. Y Rodeiro, D. (2006). Eje 1: Enseñanza-Aprendizaje, en Las TIC en el Sistema Universitario Español (2006): Un análisis estratégico. Dres.: Barro Ameneiro, S. y Burillo López, P. Madrid: Conferencia de Rectores de las Universidades Españolas.

SOCIEDAD ESPAÑOLA DE FÍSICA MÉDICA (2004): La Física Médica: "una profesión para la ciencias de la salud". Madrid: SEFM.

Tabakov, S (2005). Guest Editor of the special issue on "e-Learning in Medical Engineering and Physics”. Elsevier's Journal of Medical Engineering and Physics, 27.

- (2008): e-Learning development in medical physics and engineering. Biomed Imaging Interv J, 4:e27, 1-5. Available online at http://www.biij.org/2008/1/E27

Vázquez Mata, G. (2007). Modelos, estrategias y tendencias en España de la simulación en Medicina, en Forum Míriam Friedman. Las simulaciones en Educación Médica. Educación Médica, 10: 147-148.

Vázquez, G. Y Blanco, C. (2006). e.Learning, una necesidad inaplazable. Educación Médica, 9 (Supl. 2): 5.

Vázquez, G., Riley, D. Y Cuervo, R.L. (2006). La investigación en la formación "WebLearning”. Educación Médica, 9 (Supl. 2): 46-50.

Vázquez Mata, G. (2008). Realidad virtual y simulación en el entrenamiento de los estudiantes de medicina. Educación Médica, 11 (Supl.1): 529-531.

Vermandel, M., Betrouni, N., Rousseau, J. Y Dubois, P. (2007). Intervencional therapy procedures assisted by medical imaging and simulation. The experience of U 703 Inserm (Lille - France). Cell Mol Biol , 53: 1-10.

Francisco Javier Cabrero Fraile, José Miguel Sánchez Llorente, Ana B. Sánchez García, Javier Borrajo Sánchez, Ma José Rodríguez
Conde, Marta Cabrero Hernández y Juan Antonio Juanes Méndez 


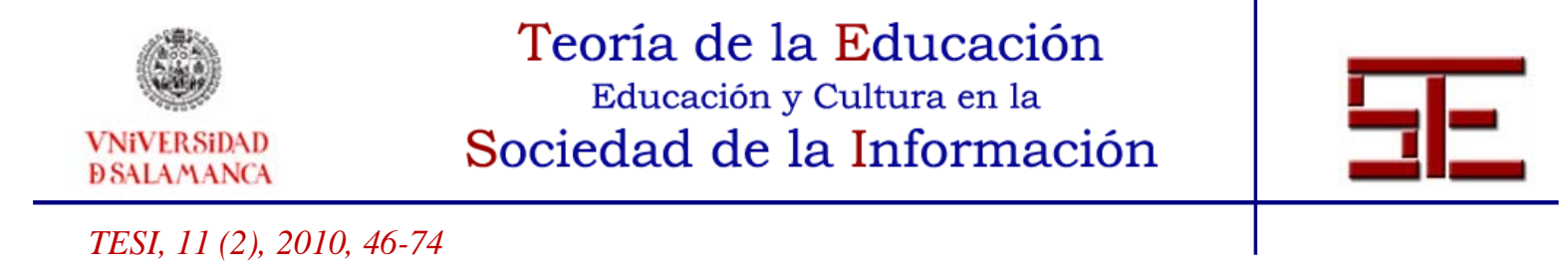

Zavala, G. Y Velarde, J.J. (2009). Estudio del aprendizaje en un curso de física universitaria usando simulaciones computacionales en la estrategia educativa. $X$ Congreso Nacional de Investigación Educativa. Veracruz (México), 21-25 de septiembre de 2009.

Ziv, A. Y Berkenstadt, H. (2008). La educación médica basada en simulaciones. Jano, 1701: 42-45.

Ziv, A., Wolpe, P., Small, S. Y Glick, S. (2003). Simulation-based medical education an ethical imperative. Academic Medicine, 78: 783-788.

\section{ENLACES DE INTERÉS}

http://phet.colorado.edu/index.php

http://www.colorado.edu./physics/2000/index.pl

http://www.enciga.org/taylor/lv.htm

http://www.isftic.mepsyd.es/profesores/asignaturas/fisica_y_quimica/

http://www.ite.educacion.es/

http://www.meet-physics.net/David-Harrison/index_spa.html

http://www.merlot.org/merlot/materials.htm?category=2736

http://www.phy.ntnu.edu.tw/ntnujava/

http://www.sc.ehu.es/sbweb/fisica_/

http://www.smete.org

http://www.sun.com/

http://www.teachersdomain.org/collection/k12/sci.phys/

Para citar el presente artículo puede utilizar la siguiente referencia:

Cabrero Fraile, F.J., Sánchez Llorente, J.M., Sánchez García, A.B., Borrejo Sánchez, J., Rodríguez Conde, Ma J., Cabrero Hernández, M. y Juanes Méndez, J.A. (2010). Simulaciones computacionales en la enseñanza de la Física Médica, en Juanes Méndez, J.A. (Coord.) Avances tecnológicos digitales en metodologías de innovación docente en el campo de las Ciencias de la Salud en España. Revista Teoría de la Educación: Educación y Cultura en la Sociedad de la Información. Vol. 11, n 2. Universidad de Salamanca, pp. 46-55 [Fecha de consulta: dd/mm/aaaa].

http://campus.usal.es/ revistas_trabajo/index.php/revistatesi/article/view/7071/7104

Francisco Javier Cabrero Fraile, José Miguel Sánchez Llorente, Ana B. Sánchez García, Javier Borrajo Sánchez, $M^{a}$ José Rodríguez Conde, Marta Cabrero Hernández y Juan Antonio Juanes Méndez 